\title{
Post Millennium Development Goals Prospect on Child Mortality in India: An Analysis Using Autoregressive Integrated Moving Averages (ARIMA) Model
}

\author{
Partha De1, Damodar Sahu ${ }^{2 *}$, Arvind Pandey' ${ }^{2}$, B. K. Gulati2, Nomita Chandhiok ${ }^{3}$, \\ Arvind Kumar Shukla ${ }^{4}$, Pavitra Mohan5 ${ }^{5}$ Raj Gautam Mitra ${ }^{5}$ \\ ${ }^{1}$ Indian Statistical Institute, Kolkata, India \\ ${ }^{2}$ National Institute of Medical Statistics, ICMR, Ansari Nagar, New Delhi, India \\ ${ }^{3}$ Indian Council of Medical Research, New Delhi, India \\ ${ }^{4}$ Subharti Medical College, Meerut, India \\ ${ }^{5}$ UNICEF, New Delhi, India \\ Email: partha@isical.ac.in, ^sahunims@gmail.com, sahuicmr@gmail.com, arvindp.nims@gmail.com,gulbk@hotmail.com, \\ n_chandhiok@hotmail.com, arvindshukla_vns@rediffmail.com, basichealthcaretrust@gmail.com, rgmitra2@hotmail.com
}

How to cite this paper: De, P., Sahu, D., Pandey, A., Gulati, B.K., Chandhiok, N., Shukla, A.K., Mohan, P. and Mitra, R.G. (2016) Post Millennium Development Goals Prospect on Child Mortality in India: An Analysis Using Autoregressive Integrated Moving Averages (ARIMA) Model. Health, 8, 1845-1872.

http://dx.doi.org/10.4236/health.2016.815176

Received: November 16, 2016

Accepted: December 26, 2016

Published: December 29, 2016

Copyright $\odot 2016$ by authors and Scientific Research Publishing Inc. This work is licensed under the Creative Commons Attribution International License (CC BY 4.0).

http://creativecommons.org/licenses/by/4.0/

\section{Abstract}

Background \& Objectives: Sustainable Development Goals (SDGs) are set up as a part of the Post Millennium Development Goals (MDGs). Then it becomes essential to review the achievement of the MDGs in India and lessons learned to incorporate into the SDGs. The present study reviews and predicts different components of under-five mortality rate beyond 2015 to assess the present situation and to determine the future possibilities of achieving the new targets for SDGs in India. Data and Methods: It uses available time series data on different components of U5MR from the India's Sample Registration System (SRS). Autoregressive Integrated Moving Averages (ARIMA) model has been taken as the method of time series analysis to forecast the mortality rates beyond 2015. Results: There is a consistent pattern of faster decline in the under-five mortality compared with the neonatal mortality rate across all major states in India although neonatal mortality contributes largest share in under-five mortality. Again, share of neonatal death among under-five death is increasing steadily over the future projected years. This indicates very slow progress of reduction in neonatal mortality. Stimulating efforts with new intervention programmes will be needed to focus more on lowering neonatal mortality particularly in rural India.

\section{Keywords}

Under-Five Mortality, Infant Mortality, Neonatal Mortality, Sustainable Development 
Goals, Post-2015 Development Agenda, ARIMA Model, Mortality Projection

\section{Introduction}

The under-five mortality is considered as a key indicator of child well-being and also a broad indicator of social and economic progress of a nation. The United Nations' Millennium Development Goal 4 (MDG-4) had declared it as one of the main indicators for assessing and monitoring progress in child health. The target of the MDG-4 was to reduce the under-five mortality rate (U5MR) by two-thirds between 1990 and 2015. India being the signatory of the Millennium Declaration of the UN Millennium Summit of 2000 was committed to the achievement of Millennium Development Goals (MDGs) by the year 2015. It aimed at reducing under-five mortality rate (U5MR) from 116 deaths per thousand live births in 1990 to 42 in 2015 and infant mortality rate (IMR) from 83 deaths per thousand live births in 1990 to 27 in 2015 [1]. The world has completed the final year 2015 of the Millennium Development Goals. The number of under-five deaths worldwide has declined from nearly 12.7 million in 1990 to 5.9 million in 2015 [2]. As a result, the global U5MR has dropped from almost 91 deaths per 1000 live births in 1990 to 43 in 2015 [2]. However, despite these achievements, over 17,000 children still die each day before their fifth birthday from often preventable causes like, pneumonia, diarrhea, malaria and complications and infections during the new-born period. Forty-four per cent of under-five deaths now occur in the first month of life [2].

Again, this progress is not equally distributed at national and sub-national levels. An estimate of U5MR for different countries at the global level is periodically published by UNICEF in its publication titled "State of the World's Children" and ranks the countries as per the U5MR estimates. According to the latest report, Southern Asia region has significant variations in under-five mortality. For example, Bangladesh with U5MR of 41 per 1000 live births is ranked 60, Nepal with U5MR of 40 being ranked 61, Bhutan with U5MR of 36 being ranked 67, Pakistan with U5MR of 86 being ranked 23, Sri Lanka with U5MR of 10 being ranked 134, Iran with U5MR of 17 being ranked 100 and India with U5MR of 53 per 1000 live births ranked at 47 [3].

There is no doubt that India has experienced considerable reductions in the underfive (U5MR), infant (IMR) and neo-natal (NNMR) mortalities during the period of 1990 to 2015. For example, U5MR was declined from 126 per 1000 live births in 1990 to 48 in 2015. Similarly, IMR was declined from 88 in 1990 to 38 in 2015 and NNMR was also declined from 57 in 1990 to 28 in 2015 [2]. Despite these gains, India was unsuccessful in achieving the MDG 4 targets. Another serious concern is that, the declines in the above mortality rates across the country are heterogeneous and unequally distributed among states. For example, during the year 2013, the lowest U5MR was observed in Kerala (12) followed by Tamil Nadu (23), Delhi (26) and Maharashtra (26). On the other hand, the highest U5MR was recorded in Assam (73), followed by Madhya Pradesh (69), Orissa (66) and Uttar Pradesh (64). The other states like, Rajasthan 
(57), Bihar (54), and Chhattisgarh (53) had U5MR higher than the national level estimate (49) [4]. Similar observations may be pointed out in case of IMR at the States level. Among the bigger States, IMR is lowest in Kerala (12), followed by Tamil Nadu (21), Delhi (24) and Maharashtra (26). The highest IMR is experienced by the states like, Madhya Pradesh (54), Assam (54), Odisha (51), Uttar Pradesh (50) and Rajasthan (47). The remaining important states namely, Chhattisgarh (46), Bihar (42) and Haryana (41) recorded IMR higher than the national level estimate of 40 [4]. However, an overall reduction in U5MR and IMR of nearly 60\% and 50\% happened during 1990 to 2013 respectively indicating a remarkable decline in the recent past. Besides, along with the interstate, significant intra-state (by residence) variations exist in U5MR and its components in India. In this country, the rural areas experienced high U5MR compared to the urban areas. As per the 2013 SRS data of India, the U5MR in rural and urban areas are 55 and 29 deaths per 1000 live births respectively. During 2009-13, the rural-urban gap in U5MR declined marginally; however, the urban U5MR continued to be lower than rural U5MR by more than $40 \%$ of the corresponding rural status [4]. The same data reveals that, IMR in the rural areas continues to be at a much higher level than the urban IMR (rural and urban IMRs are 44 and 27 respectively). Although the rural urban gap is decreasing over time, but a significant difference always exists [4].

Now, the assessments of the present situation based on the light of MDGs are essential and it also becomes important to earmark the new development agenda for the future years. That is why United Nations (UN) has prepared new goals for development progress to guide international efforts over the next 15 years. The Post MDG Agenda has already been placed in the assembly in New York in September 2015 and consequently a new set of Sustainable Development Goals (SDGs) are being adopted by world leaders. To promote long and healthy lives among the children and also to progress towards wiping out preventable child mortality, target has been fixed to reach the child mortality by 2030 to half its level in 2010 . More specifically, by 2030 , all countries should aiming to reduce neonatal mortality to at least as low as 12 per 1000 live births and under-5 mortality to at least as low as 25 per 1000 live births [5].

It is then prudent to forecast the future under-five mortality and its components for understanding our immediate tasks in reduction of such deaths, so that finally we can achieve the future SDG targets of child mortality in India and its states. There has been hardly any attempt in this direction in recent past for making an assessment about India's present position and possibilities of reaching the future target of child mortality fixed by the Sustainable Development Goal 3 under Post-2015 development Agenda. The available past attempts mainly concentrated on to find out the possibilities of attaining MDG goals in case of under-five mortality. So the entire focus was restricted up to 2015. Those approaches have broadly utilized simple trend analysis of individual components of U5MR at the global level [4] [5] [6] and country level, e.g. Bangladesh [7], Sri Lanka [8], and India [9]. In present study a time series analysis has been carried out by applying Autoregressive Integrated Moving Averages (ARIMA) model examining the forecast of the future levels of neonatal, infant and under five mortality rates in 
India and its states. Along with under-five and infant mortalities, the trend in the level of neonatal mortality (mortality within first 28 days) is also examined in view of highest numbers of deaths occurring in India in the first four weeks of human life [10]. The study is carried out with a view to refine understanding of the future course of mortality among children evaluating country's present status in achieving the future Sustainable Development Goal 3 (SDG-3).

\section{Data \& Methods}

The time series data (1981-2013) from the Sample Registration System [4] (Registrar General, India)on neonatal, infant and under-five mortalities in rural and urban areas of India and its 16 major states, are used for the projection of future mortality in case of neonatal, infant and under-five. For the state of Jammu and Kashmir, systematic time series data are not available and hence projection is not done for the state. Three states namely, Chhattisgarh, Jharkhand and Uttaranchal are included in their mother states like, Madhya Pradesh, Bihar and Uttar Pradesh respectively to prepare the time series data for a long period of 33 years.

Since, the data are used for the analysis from a common source, which is SRS, we do not require any specific assumption about the behavior of data series, which are required, if data points belong to different sources. The other source of data for infant and child mortality in India is National Family Health Survey (NFHS) but with limited three rounds and the fourth round of survey is in vogue. The level and pattern of infant and child mortality in both the surveys are found to be similar [11]. The major challenge in time series modeling is posed by the fact that the error residuals are correlated with their own lagged values violating the standard assumption of regression theory that disturbances are not correlated. We adopt here autoregressive integrated moving average (ARIMA) time series model to forecast the neonatal, infant and under-five mortalities for the period of 2015-2030 based on the data values taken from SRS for the period 1981-2013. The ARIMA model takes care of this serial correlation [12] by taking into account the historical fluctuations, trends, seasonality, cycles, prediction errors and non-stationarity of the data. The model helps to improve accuracy in forecasting [13]. The ARIMA modeling approach is pioneered by Box and Jenkins [14] and has been widely used in forecasting of different social and economic real life variables. The ARIMA time series models are preferred over simple and intuitive methods to obtain estimates and future projections by smoothing of data followed by extrapolation. This is due to the fact that simple trend analysis assumes that the past trends uninterruptedly continue in the future which cannot be assumed while modeling the demographic indicators because of very high uncertainty [15]. Furthermore, a time series model could be either stationary or non-stationary. The stationary model assumes that the process remains in equilibrium with a constant mean whereas in real life, empirical time series do not have a fixed mean. Hence, models are developed to describe homogeneous non-stationary behavior by supposing some suitable differences of the process to be stationary [16]. This type of model is known as non-stationary model and denoted as 
ARIMA (p, d, q) where, $\mathrm{p}, \mathrm{d}$ and $\mathrm{q}$ denote orders of auto-regression, integration (differencing) and moving average respectively. Here, parameter "p" provides information concerning the order of structural dependence existent between adjacent observations, indicating the existence of autocorrelation. The parameter " $d$ " denotes the number of times the series must be differentiated in order to become stationary and the parameter " $q$ " indicates the number of moving average terms. Thus, we have to identify whether the applied model is autoregressive (AR), moving averages (MA), autoregressive moving averages (ARMA) or auto regressive integrated moving averages (ARIMA).

The SRS data on neonatal, infant and under-five mortalities for the period of 1981-2013 were found to be non-stationary. They were therefore converted to stationary series by first transforming by taking its natural logarithm and then by applying successive differencing of the transformed series. To obtain the predicted values, the projected values were derived by taking antilogarithm. To build up the model for each state and India, whether the series is stationary or not is judged by estimating sample autocorrelation function (ACF) and partial autocorrelation functions (PACF). Autocorrelation refers to the way the observations in a time series was related to each other and is measured by the simple correlation between current observation $\left(\mathrm{Y}_{\mathrm{t}}\right)$ and observations from " $\mathrm{p}$ " periods before the current one $\left(\mathrm{Y}_{\mathrm{t}-\mathrm{p}}\right)$. Partial autocorrelations are used to measure the degree of association between data points $Y_{t}$ and $Y_{t-p}$ when the $Y$-effects at other time lags $1,2,3 \ldots \mathrm{p}-1$ are removed. The stationarity is verified by applying an Augmented Dickey Fuller test. So by differencing the data series and testing by ADF, stationary model is determined, selection of the model for any state or India may vary according to the nature of time series data included in the analysis.

\section{Results}

In the present study, projection of NNMR, IMR and U5MR of major Indian states including India as a whole up to the year 2030 are presented. To identify the intra-state variations based on residence type, rural and urban areas of each state are projected separately along with the entire state under consideration. This projection exercise is done for a duration of 15 years, starting from 2015 and ending with 2030, which is actually the target year for SDG goals under Post-2015 Development Agenda. However, mortality projections are restricted for a small period because different important parameters related with socio-economic and demographic conditions in the country may change over time and affect the projected values. The Tables 1 (a)-(c) are presented for infant mortality. Similarly, Tables 2(a)-(c) are included for under-five mortality. Lastly, Tables 3(a)-(c) are created for neonatal mortality. All the projected values in the tables are based on different fitted ARIMA ( $p, d, q)$ models. The predicted values from 2015 to 2030 of the Box-Jenkins results are illustrated in the tables. The forecasts and 95\% forecast confidence intervals for IMR are shown in Figures 1(a)-(c) for the total, rural and urban areas of India respectively. Similar Figures 2(a)-(c) and Figures 3 (a)-(c) are also presented for U5MR and NNMR respectively.

It has already been mentioned that SDG3 target has to be fixed to reach the child 
Table 1. (a) Predicted values of infant mortality rates (Total) and associated 95\% confidence intervals using ARIMA model by states and India, 2015-2030; (b) Predicted values of infant mortality rates (Rural) and associated 95\% confidence intervals using ARIMA model by states and India, 2015-2030; (c) Predicted values of infant mortality rates (Urban) and associated 95\% confidence intervals using ARIMA model by states and India, 2015-2030.

(a)

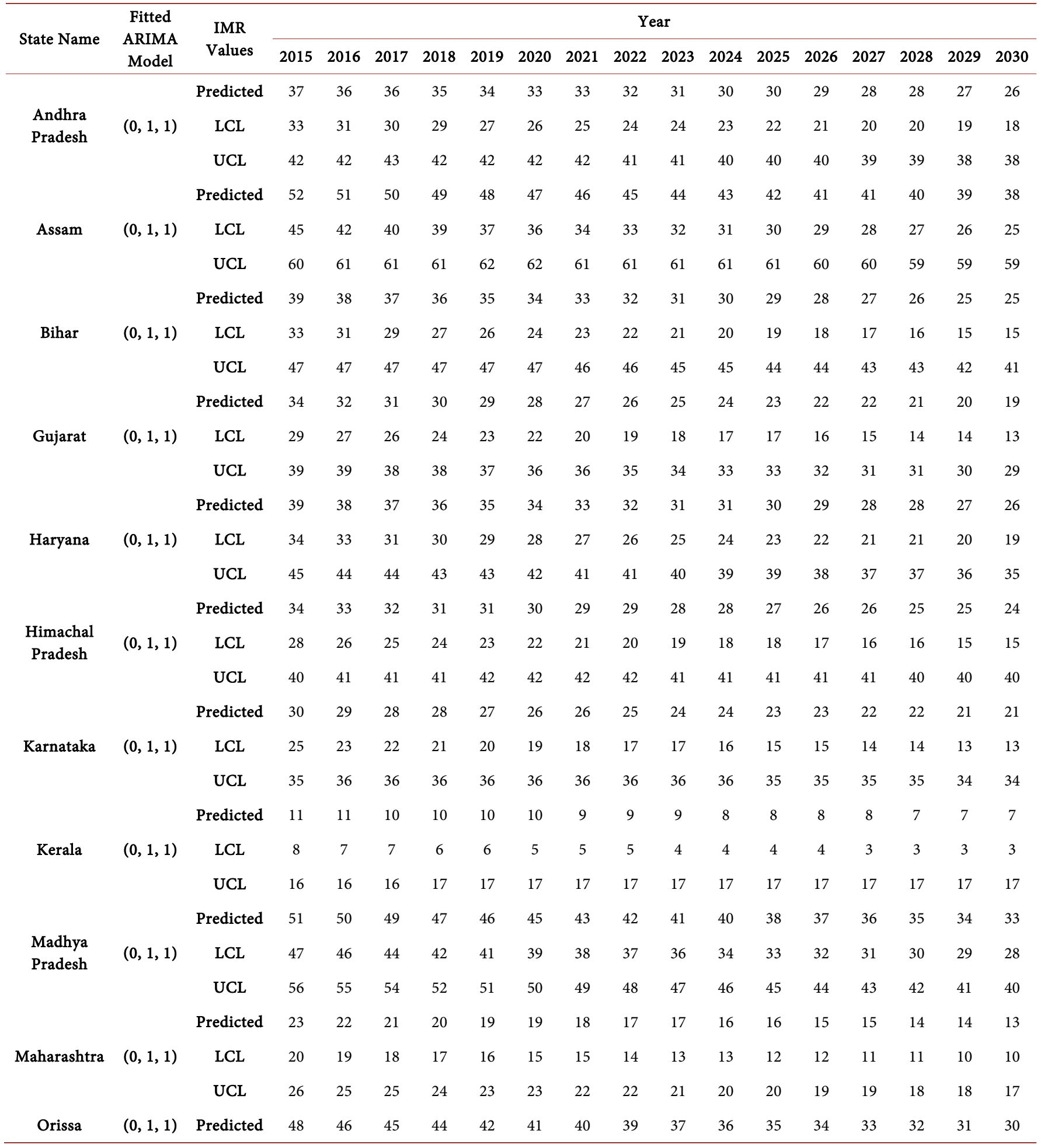


Continued

\begin{tabular}{|c|c|c|c|c|c|c|c|c|c|c|c|c|c|c|c|c|c|c|}
\hline & & LCL & 44 & 41 & 39 & 38 & 36 & 34 & 33 & 31 & 30 & 29 & 28 & 27 & 26 & 25 & 24 & 23 \\
\hline & & Predicted & 24 & 24 & 23 & 22 & 21 & 21 & 20 & 19 & 19 & 18 & 17 & 17 & 16 & 16 & 15 & 15 \\
\hline \multirow[t]{2}{*}{ Punjab } & $(0,1,1)$ & LCL & 21 & 20 & 19 & 18 & 17 & 16 & 15 & 14 & 14 & 13 & 12 & 12 & 11 & 11 & 10 & 10 \\
\hline & & UCL & 28 & 28 & 28 & 28 & 27 & 27 & 26 & 26 & 25 & 25 & 24 & 24 & 23 & 23 & 22 & 22 \\
\hline \multirow[t]{3}{*}{ Rajasthan } & $(0,1,1)$ & LCL & 38 & 36 & 35 & 33 & 32 & 30 & 29 & 28 & 27 & 26 & 25 & 24 & 23 & 22 & 21 & 21 \\
\hline & & UCL & 53 & 53 & 53 & 53 & 53 & 53 & 52 & 52 & 52 & 51 & 51 & 50 & 50 & 49 & 49 & 48 \\
\hline & & Predicted & 19 & 18 & 18 & 17 & 16 & 15 & 15 & 14 & 14 & 13 & 12 & 12 & 11 & 11 & 10 & 10 \\
\hline Tamil Nadu & $(0,1,1)$ & LCL & 16 & 15 & 14 & 13 & 12 & 12 & 11 & 10 & 10 & 9 & 8 & 8 & 8 & 7 & 7 & 6 \\
\hline \multirow[t]{3}{*}{ Uttar Pradesh } & $(0,1,1)$ & LCL & 41 & 38 & 36 & 34 & 32 & 30 & 28 & 27 & 25 & 24 & 23 & 22 & 21 & 20 & 19 & 18 \\
\hline & & UCL & 53 & 53 & 52 & 52 & 51 & 50 & 50 & 49 & 48 & 47 & 46 & 45 & 44 & 43 & 42 & 41 \\
\hline & & Predicted & 27 & 27 & 26 & 26 & 25 & 24 & 23 & 22 & 22 & 21 & 20 & 20 & 19 & 18 & 18 & 17 \\
\hline \multirow[t]{3}{*}{ West Bengal } & $(0,1,1)$ & LCL & 25 & 24 & 23 & 22 & 21 & 21 & 20 & 19 & 18 & 18 & 17 & 16 & 16 & 15 & 14 & 14 \\
\hline & & UCL & 32 & 31 & 30 & 30 & 29 & 28 & 27 & 27 & 26 & 25 & 24 & 24 & 23 & 22 & 22 & 21 \\
\hline & & Predicted & 38 & 36 & 35 & 34 & 33 & 32 & 31 & 30 & 29 & 28 & 28 & 27 & 26 & 25 & 24 & 24 \\
\hline India & $(0,1,1)$ & LCL & 35 & 33 & 31 & 30 & 29 & 28 & 26 & 25 & 24 & 23 & 22 & 22 & 21 & 20 & 19 & 18 \\
\hline
\end{tabular}

NOTE: ARIMA-Autoregressive integrated moving averages; LCL-lower confidence level; UCL-upper confidence level. The states Bihar, Uttar Pradesh and Madhya Pradesh include Jharkhand, Uttarakhand and Chhattisgarh respectively. ARIMA ( $p, d, q)-p, d$ and q denote orders of auto-regression, integration (differencing) and moving average respectively.

(b)

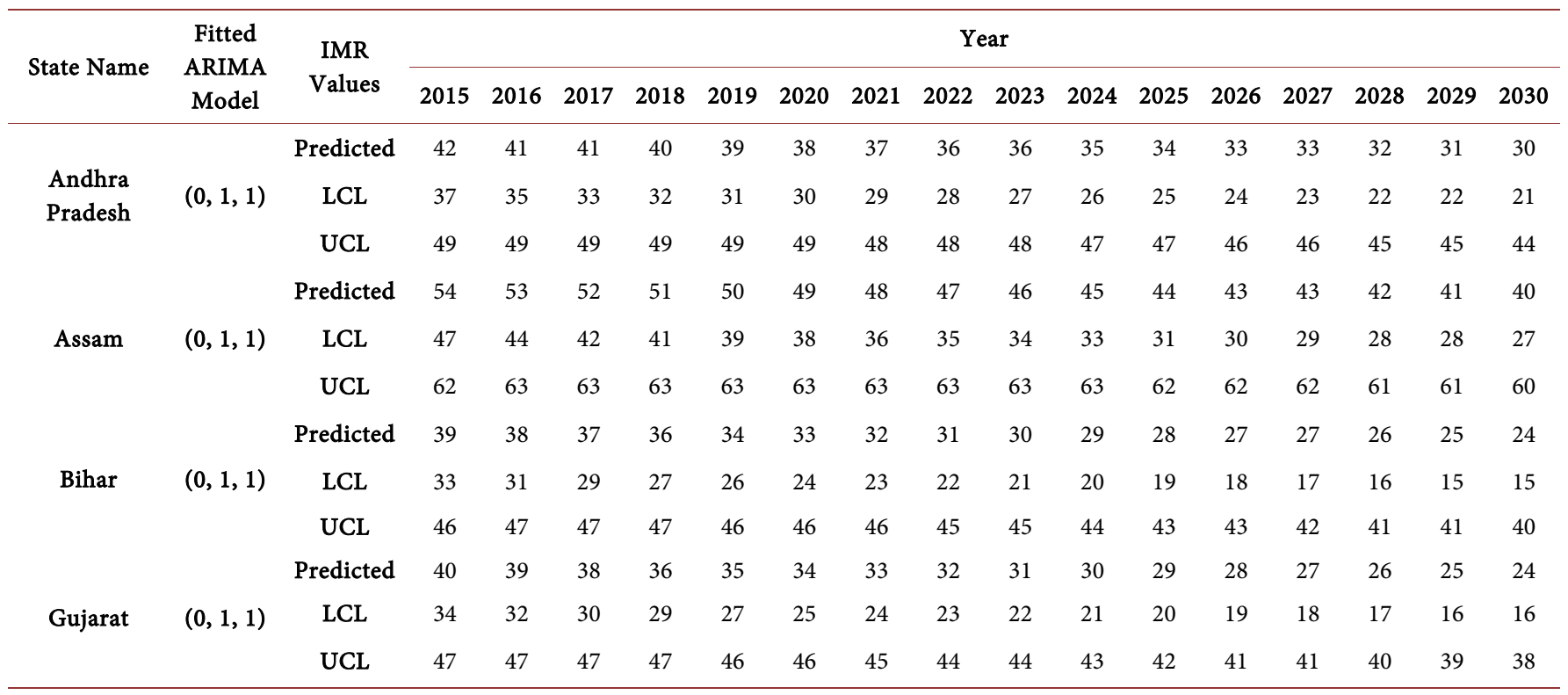


Continued

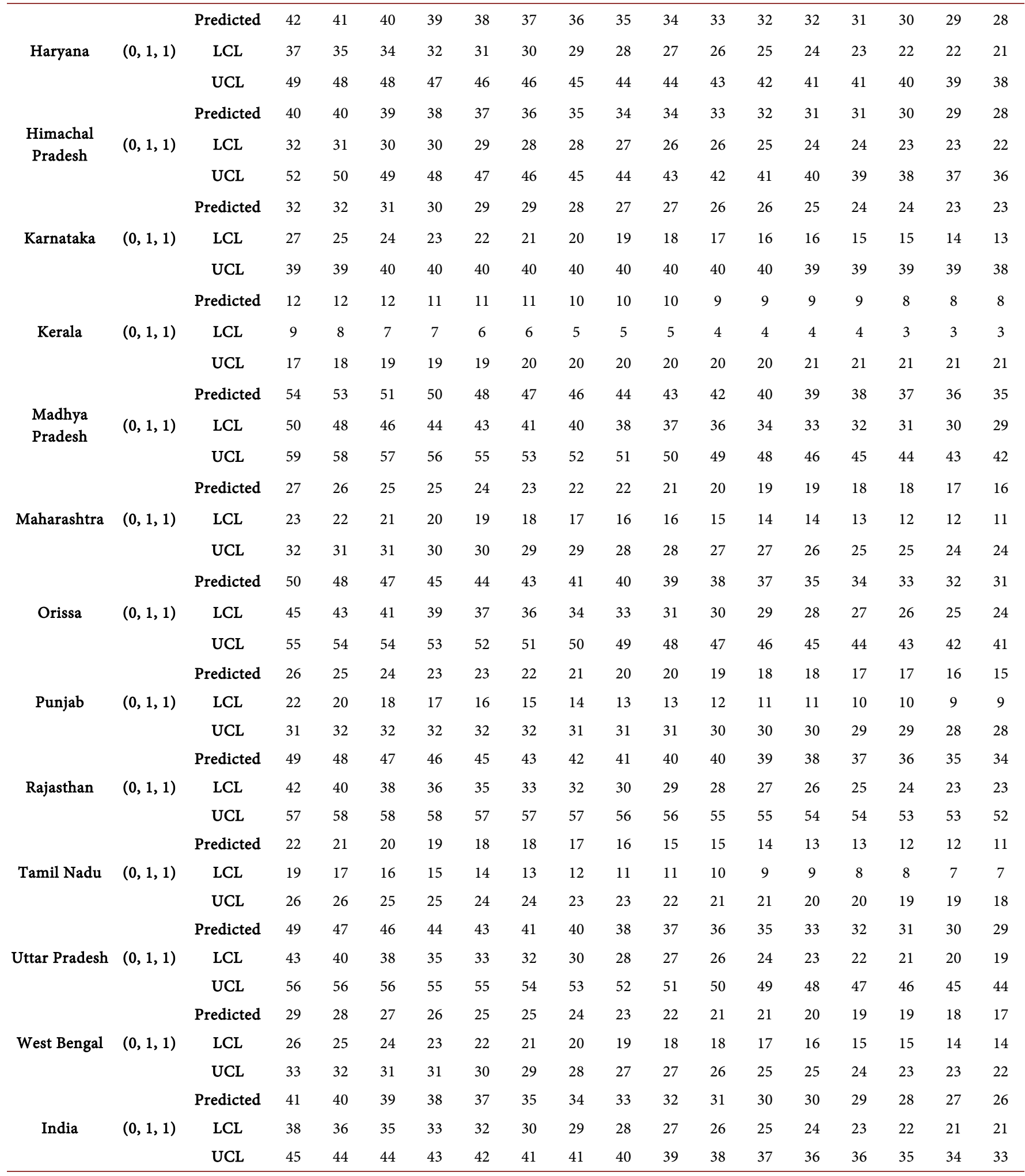

NOTE: ARIMA-Autoregressive integrated moving averages; LCL-lower confidence level; UCL—upper confidence level. The states Bihar, Uttar Pradesh and Madhya Pradesh include Jharkhand, Uttarakhand and Chhattisgarh respectively. ARIMA ( $, \mathrm{d}, \mathrm{q}$ ) - p, d and q denote orders of auto-regression, integration (differencing) and moving average respectively. 
(c)

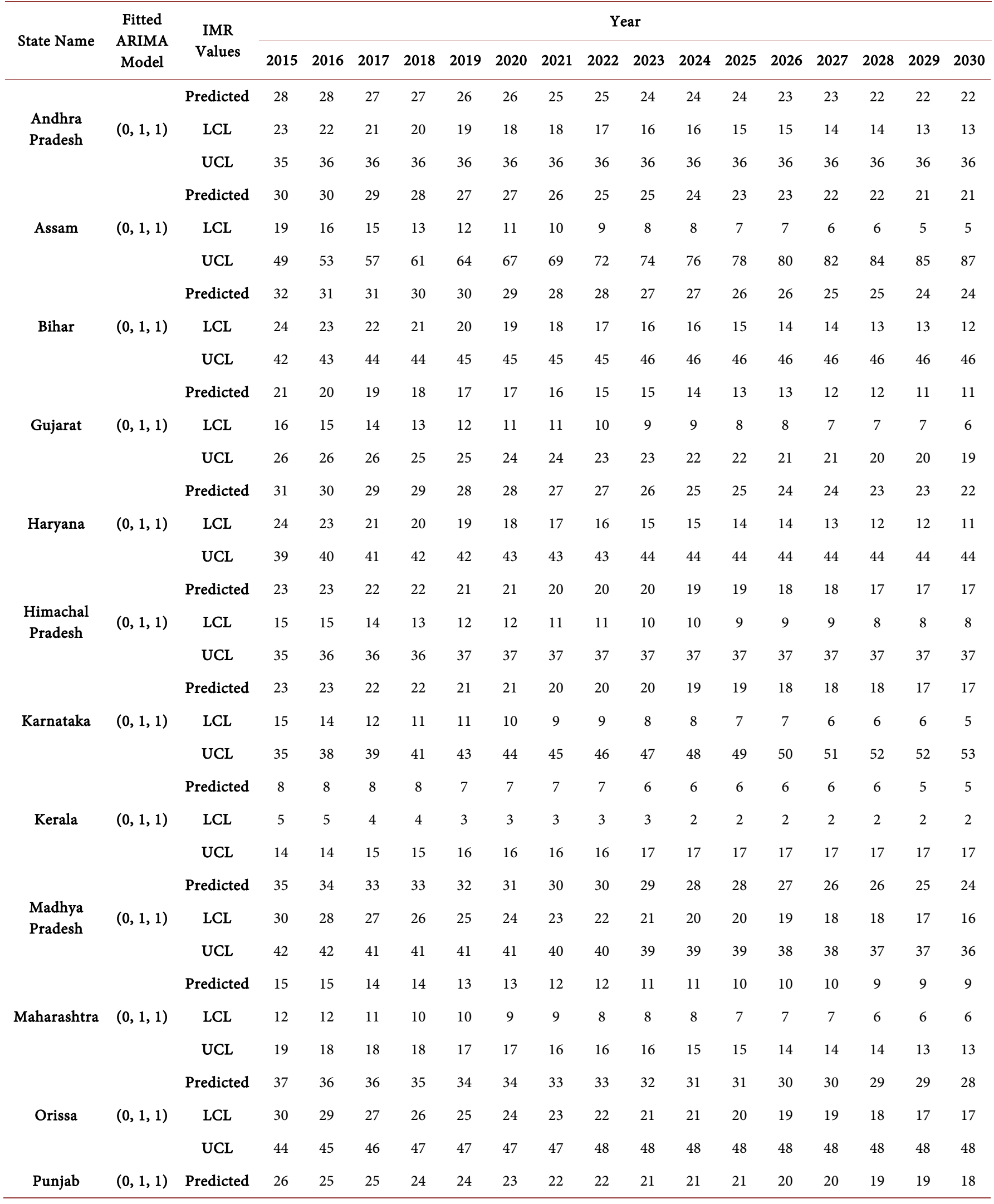


Continued

\begin{tabular}{|c|c|c|c|c|c|c|c|c|c|c|c|c|c|c|c|c|c|c|}
\hline & & LCL & 20 & 19 & 19 & 18 & 18 & 18 & 17 & 17 & 16 & 16 & 16 & 15 & 15 & 15 & 14 & 14 \\
\hline & & Predicted & 29 & 28 & 28 & 27 & 26 & 26 & 25 & 25 & 24 & 24 & 23 & 23 & 22 & 22 & 21 & 21 \\
\hline \multirow[t]{2}{*}{ Rajasthan } & $(0,1,1)$ & LCL & 22 & 21 & 19 & 18 & 17 & 17 & 16 & 15 & 14 & 14 & 13 & 13 & 12 & 12 & 11 & 11 \\
\hline & & UCL & 38 & 38 & 39 & 39 & 40 & 40 & 40 & 41 & 41 & 41 & 41 & 41 & 41 & 41 & 41 & 40 \\
\hline \multirow[t]{3}{*}{ Tamil Nadu } & $(0,1,1)$ & LCL & 13 & 12 & 11 & 10 & 9 & 9 & 8 & 8 & 7 & 7 & 6 & 6 & 6 & 5 & 5 & 5 \\
\hline & & UCL & 20 & 20 & 20 & 20 & 20 & 20 & 20 & 20 & 20 & 19 & 19 & 19 & 19 & 18 & 18 & 18 \\
\hline & & Predicted & 37 & 36 & 34 & 33 & 32 & 32 & 31 & 30 & 29 & 28 & 27 & 26 & 26 & 25 & 24 & 23 \\
\hline Uttar Pradesh & $(0,1,1)$ & LCL & 32 & 31 & 30 & 29 & 28 & 27 & 26 & 25 & 24 & 23 & 23 & 22 & 21 & 20 & 20 & 19 \\
\hline \multirow[t]{3}{*}{ West Bengal } & $(0,1,1)$ & LCL & 19 & 19 & 18 & 18 & 17 & 17 & 16 & 16 & 15 & 15 & 15 & 14 & 14 & 13 & 13 & 13 \\
\hline & & UCL & 30 & 30 & 30 & 29 & 29 & 28 & 28 & 27 & 27 & 26 & 26 & 26 & 25 & 25 & 24 & 24 \\
\hline & & Predicted & 26 & 25 & 24 & 24 & 23 & 23 & 22 & 21 & 21 & 20 & 20 & 19 & 19 & 18 & 18 & 17 \\
\hline \multirow[t]{2}{*}{ India } & $(0,1,1)$ & LCL & 23 & 22 & 21 & 20 & 19 & 19 & 18 & 17 & 17 & 16 & 16 & 15 & 15 & 14 & 14 & 13 \\
\hline & & UCL & 29 & 29 & 28 & 28 & 28 & 27 & 27 & 26 & 26 & 25 & 25 & 24 & 24 & 23 & 23 & 22 \\
\hline
\end{tabular}

NOTE: ARIMA-Autoregressive integrated moving averages; LCL-lower confidence level; UCL-upper confidence level. The states Bihar, Uttar Pradesh and Madhya Pradesh include Jharkhand, Uttarakhand and Chhattisgarh respectively. ARIMA ( $p, d, q)-p, d$ and q denote orders of auto-regression, integration (differencing) and moving average respectively.

Table 2. (a) Predicted values of under-five mortality rates (Total) and associated $95 \%$ confidence intervals using ARIMA model by states and India, 2015-2030; (b) Predicted values of under-five mortality rates (Rural) and associated 95\% confidence intervals using ARIMA model by states and India, 2015-2030; (c) Predicted values of under-five mortality rates (Urban) and associated 95\% confidence intervals using ARIMA model by states and India, 2015-2030.

(a)

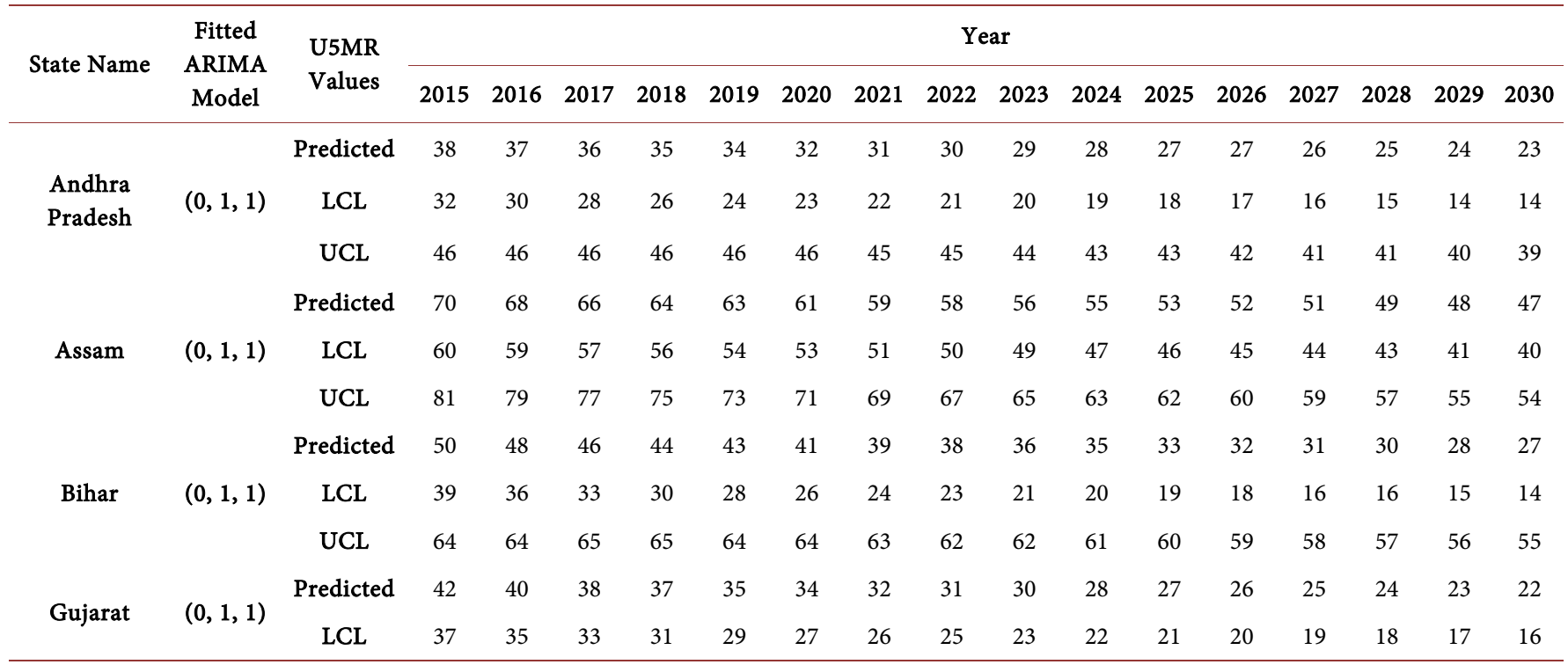




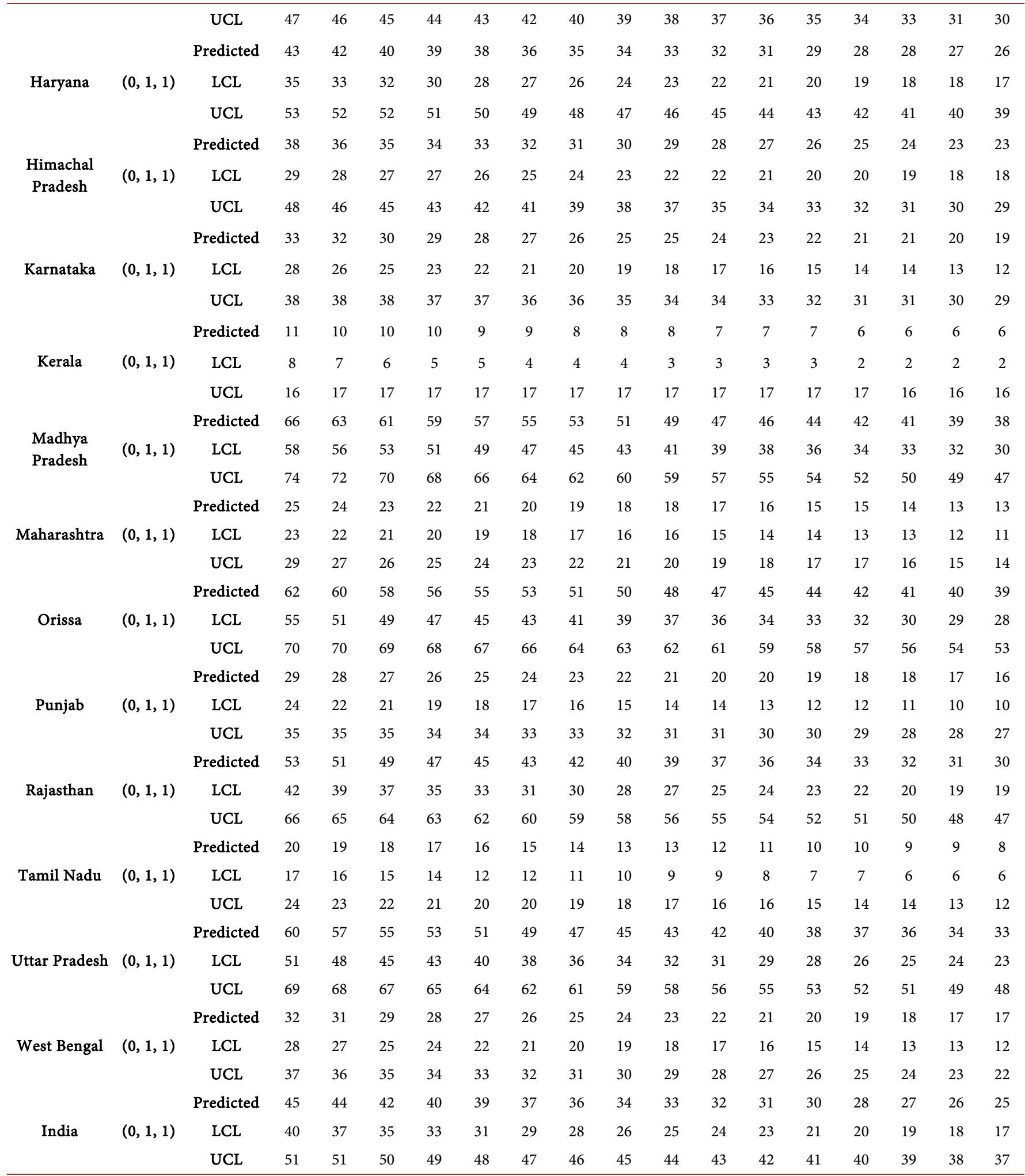

NOTE: ARIMA-Autoregressive integrated moving averages; LCL-lower confidence level; UCL-upper confidence level. The states Bihar, Uttar Pradesh and Madhya Pradesh include Jharkhand, Uttarakhand and Chhattisgarh respectively. ARIMA ( $, d, q)-p, d$ and q denote orders of auto-regression, integration (differencing) and moving average respectively. 
(b)

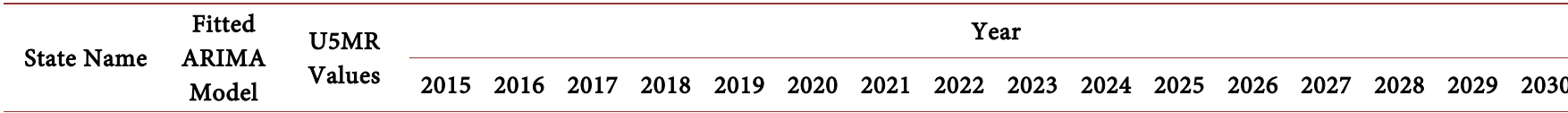

$\begin{array}{lllllllllllllllll}\text { Predicted } & 43 & 42 & 40 & 39 & 38 & 36 & 35 & 34 & 33 & 32 & 31 & 30 & 29 & 28 & 27 & 26\end{array}$

Andhra

Pradesh

$(0,1,1)$

LC

$\begin{array}{llllll}\text { Predicted } & 74 & 72 & 70 & 69 & 67\end{array}$

Assam

$(0,1,1)$

LC

$\begin{array}{llll}\text { UCL } & 87 & 85 & 83\end{array}$

Predicted $\quad 52 \quad 49 \quad 47$

Bihar

$(0,1,1)$

$\begin{array}{lllll}\text { LCL } & 40 & 36 & 33 & 31\end{array}$

$\begin{array}{lllllllllllllllll}\text { UCL } & 66 & 67 & 67 & 67 & 67 & 66 & 66 & 65 & 64 & 63 & 62 & 61 & 60 & 59 & 58 & 57\end{array}$

$\begin{array}{lllllllllllllllll}\text { Predicted } & 49 & 47 & 45 & 43 & 42 & 40 & 38 & 37 & 35 & 34 & 32 & 31 & 30 & 29 & 28 & 26\end{array}$

Gujarat

Pradesh

$(0,1,1)$

Predicted $47 \quad 45 \quad 44 \quad 42$

(c)

$\begin{array}{lllllllllllllllll}\text { UCL } & 59 & 58 & 57 & 56 & 55 & 54 & 52 & 51 & 50 & 49 & 48 & 47 & 46 & 44 & 43 & 42\end{array}$

$\begin{array}{lllllllllllllllll} & 38 & 37 & 36 & 34 & 33 & 32 & 31 & 30 & 29 & 28 & 27 & 26 & 25 & 24 & 24 & 23\end{array}$

$\begin{array}{lllllllllllllllll}\text { UCL } & 49 & 48 & 46 & 44 & 43 & 41 & 40 & 39 & 37 & 36 & 35 & 34 & 33 & 31 & 30 & 29\end{array}$

$\begin{array}{llllllllllllllllll}\text { Predicted } & 35 & 34 & 33 & 32 & 31 & 29 & 28 & 27 & 26 & 25 & 24 & 24 & 23 & 22 & 21 & 20\end{array}$

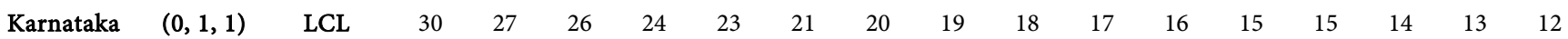

$\begin{array}{lllllllllllllllll}\text { UCL } & 42 & 42 & 42 & 42 & 41 & 40 & 40 & 39 & 38 & 38 & 37 & 36 & 35 & 35 & 34 & 33\end{array}$

$\begin{array}{lllllllllllllllll}\text { Predicted } & 12 & 11 & 11 & 10 & 10 & 9 & 9 & 9 & 8 & 8 & 8 & 7 & 7 & 7 & 6 & 6\end{array}$

$\begin{array}{llllllllllllllllll}\text { Kerala } & (0,1,1) & \text { LCL } & 8 & 7 & 6 & 6 & 5 & 5 & 4 & 4 & 4 & 3 & 3 & 3 & 3 & 3 & 2\end{array}$

$\begin{array}{lllllllllllllllll}\text { UCL } & 18 & 18 & 18 & 18 & 19 & 19 & 19 & 18 & 18 & 18 & 18 & 18 & 18 & 17 & 17 & 17\end{array}$

$\begin{array}{lllllllllllllllll}\text { Predicted } & 70 & 68 & 65 & 63 & 61 & 58 & 56 & 54 & 52 & 50 & 48 & 46 & 45 & 43 & 41 & 40\end{array}$

Madhya

Pradesh

$\begin{array}{llllllllllllllllll}(0,1,1) & \text { LCL } & 63 & 60 & 57 & 54 & 52 & 49 & 47 & 45 & 43 & 41 & 39 & 38 & 36 & 34 & 33 & 31\end{array}$

$\begin{array}{lllllllllllllllll}\text { UCL } & 79 & 77 & 75 & 73 & 71 & 69 & 67 & 65 & 63 & 61 & 59 & 57 & 56 & 54 & 52 & 51\end{array}$

$\begin{array}{lllllllllllllllll}\text { Predicted } & 30 & 29 & 27 & 26 & 25 & 24 & 23 & 22 & 21 & 20 & 19 & 18 & 17 & 17 & 16 & 15\end{array}$

$(0,1,1)$

$\begin{array}{lllllllllllllllll}\text { UCL } & 34 & 33 & 31 & 30 & 29 & 27 & 26 & 25 & 24 & 23 & 22 & 21 & 20 & 19 & 18 & 17\end{array}$

$\begin{array}{lllllllllllllllll}\text { Predicted } & 66 & 64 & 62 & 60 & 58 & 56 & 54 & 52 & 51 & 49 & 48 & 46 & 45 & 43 & 42 & 41\end{array}$

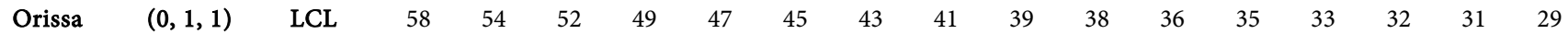

$\begin{array}{lllllllllllllllll}\text { UCL } & 75 & 74 & 73 & 72 & 71 & 69 & 68 & 67 & 66 & 64 & 63 & 62 & 60 & 59 & 58 & 56\end{array}$

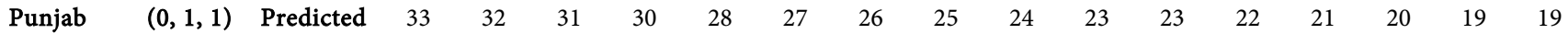


Continued

\begin{tabular}{|c|c|c|c|c|c|c|c|c|c|c|c|c|c|c|c|c|c|c|}
\hline & & LCL & 28 & 26 & 24 & 23 & 21 & 20 & 19 & 18 & 17 & 16 & 15 & 15 & 14 & 13 & 12 & 12 \\
\hline & & Predicted & 59 & 57 & 55 & 53 & 51 & 49 & 47 & 45 & 43 & 42 & 40 & 39 & 37 & 36 & 35 & 33 \\
\hline \multirow[t]{2}{*}{ Rajasthan } & $(0,1,1)$ & LCL & 47 & 44 & 41 & 39 & 37 & 35 & 33 & 31 & 29 & 28 & 26 & 25 & 24 & 23 & 21 & 20 \\
\hline & & UCL & 75 & 74 & 73 & 72 & 70 & 69 & 67 & 66 & 65 & 63 & 62 & 60 & 59 & 57 & 56 & 54 \\
\hline \multirow[t]{3}{*}{ Tamil Nadu } & $(0,1,1)$ & LCL & 20 & 18 & 17 & 15 & 14 & 13 & 12 & 11 & 10 & 10 & 9 & 8 & 8 & 7 & 7 & 6 \\
\hline & & UCL & 27 & 26 & 25 & 24 & 23 & 22 & 21 & 20 & 19 & 18 & 17 & 17 & 16 & 15 & 14 & 14 \\
\hline & & Predicted & 63 & 60 & 58 & 56 & 53 & 51 & 49 & 47 & 45 & 43 & 42 & 40 & 38 & 37 & 35 & 34 \\
\hline Uttar Pradesh & $(0,1,1)$ & LCL & 54 & 51 & 47 & 45 & 42 & 39 & 37 & 35 & 33 & 31 & 30 & 28 & 27 & 25 & 24 & 23 \\
\hline \multirow[t]{3}{*}{ West Bengal } & $(0,1,1)$ & LCL & 30 & 28 & 26 & 24 & 23 & 21 & 20 & 19 & 18 & 17 & 16 & 15 & 14 & 13 & 13 & 12 \\
\hline & & UCL & 38 & 37 & 36 & 35 & 34 & 33 & 32 & 31 & 30 & 29 & 28 & 27 & 26 & 25 & 24 & 23 \\
\hline & & Predicted & 51 & 49 & 47 & 45 & 43 & 42 & 40 & 39 & 37 & 36 & 34 & 33 & 32 & 31 & 29 & 28 \\
\hline \multirow[t]{2}{*}{ India } & $(0,1,1)$ & LCL & 45 & 42 & 40 & 37 & 35 & 33 & 31 & 30 & 28 & 27 & 25 & 24 & 23 & 22 & 21 & 20 \\
\hline & & UCL & 57 & 57 & 56 & 55 & 54 & 52 & 51 & 50 & 49 & 48 & 46 & 45 & 44 & 43 & 42 & 40 \\
\hline
\end{tabular}

NOTE: ARIMA-Autoregressive integrated moving averages; LCL—lower confidence level; UCL—upper confidence level. The states Bihar, Uttar Pradesh and Madhya Pradesh include Jharkhand, Uttarakhand and Chhattisgarh respectively. ARIMA ( $p, d, q$ ) $-p, d$ and $q$ denote orders of auto-regression, integration (differencing) and moving average respectively.

(c)

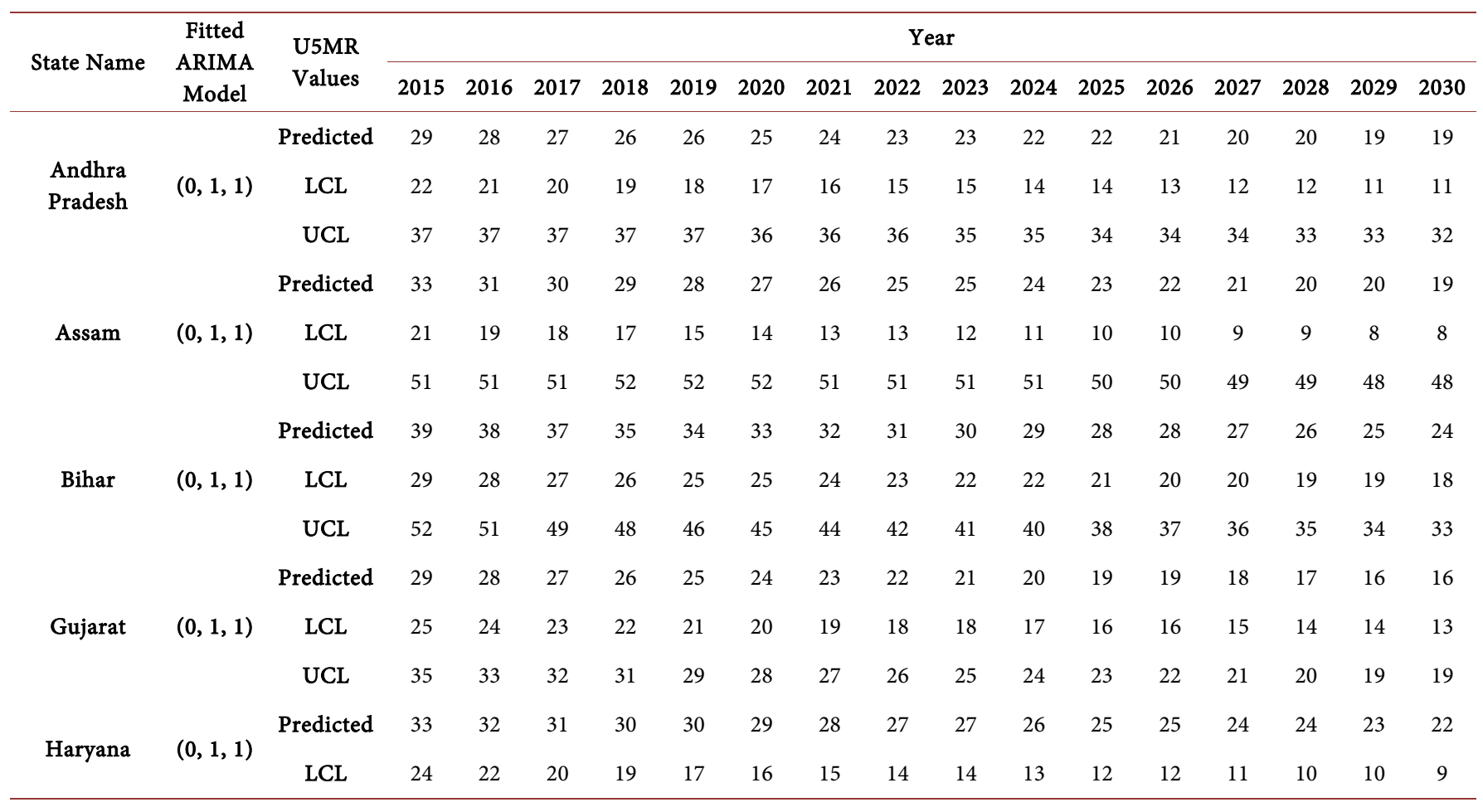




\begin{tabular}{|c|c|c|c|c|c|c|c|c|c|c|c|c|c|c|c|c|c|c|}
\hline & & UCL & 45 & 47 & 48 & 49 & 50 & 51 & 51 & 52 & 52 & 53 & 53 & 53 & 54 & 54 & 54 & 54 \\
\hline \multirow[t]{2}{*}{$\begin{array}{c}\text { Himachal } \\
\text { Pradesh }\end{array}$} & \multirow[t]{2}{*}{$(0,1,1)$} & LCL & 18 & 17 & 16 & 15 & 14 & 13 & 12 & 12 & 11 & 11 & 10 & 10 & 9 & 9 & 8 & 8 \\
\hline & & UCL & 59 & 61 & 64 & 66 & 68 & 70 & 72 & 74 & 76 & 78 & 80 & 81 & 83 & 84 & 86 & 87 \\
\hline \multirow{2}{*}{ Karnataka } & \multirow{2}{*}{$(0,1,1)$} & Predicted & 27 & 26 & 26 & 25 & 24 & 24 & 23 & 22 & 22 & 21 & 21 & 20 & 19 & 19 & 18 & 18 \\
\hline & & UCL & 38 & 39 & 40 & 40 & 41 & 41 & 41 & 41 & 41 & 41 & 41 & 41 & 41 & 41 & 41 & 41 \\
\hline \multirow{3}{*}{ Kerala } & \multirow{3}{*}{$(0,1,1)$} & Predicted & 8 & 8 & 7 & 7 & 7 & 6 & 6 & 6 & 5 & 5 & 5 & 5 & 4 & 4 & 4 & 4 \\
\hline & & LCL & 5 & 4 & 4 & 3 & 3 & 3 & 2 & 2 & 2 & 2 & 2 & 1 & 1 & 1 & 1 & 1 \\
\hline & & UCL & 14 & 15 & 15 & 15 & 16 & 16 & 16 & 16 & 16 & 16 & 16 & 16 & 16 & 16 & 16 & 15 \\
\hline \multirow{3}{*}{ Maharashtra } & \multirow{3}{*}{$(0,1,1)$} & Predicted & 18 & 17 & 17 & 16 & 15 & 15 & 14 & 13 & 13 & 12 & 12 & 11 & 11 & 10 & 10 & 9 \\
\hline & & LCL & 15 & 15 & 14 & 14 & 13 & 12 & 12 & 11 & 11 & 10 & 10 & 9 & 9 & 9 & 8 & 8 \\
\hline & & UCL & 22 & 21 & 20 & 19 & 18 & 17 & 17 & 16 & 15 & 15 & 14 & 13 & 13 & 12 & 12 & 11 \\
\hline \multirow{3}{*}{ Orissa } & \multirow{3}{*}{$(0,1,1)$} & Predicted & 37 & 35 & 34 & 33 & 32 & 31 & 29 & 28 & 27 & 26 & 26 & 25 & 24 & 23 & 22 & 21 \\
\hline & & LCL & 27 & 24 & 22 & 20 & 19 & 17 & 16 & 15 & 14 & 13 & 12 & 12 & 11 & 10 & 10 & 9 \\
\hline & & UCL & 50 & 52 & 53 & 53 & 54 & 54 & 54 & 54 & 54 & 53 & 53 & 53 & 52 & 52 & 51 & 51 \\
\hline \multirow[b]{2}{*}{ Punjab } & \multirow[b]{2}{*}{$(0,1,1)$} & Predicted & 24 & 23 & 22 & 21 & 21 & 20 & 19 & 19 & 18 & 18 & 17 & 16 & 16 & 15 & 15 & 14 \\
\hline & & LCL & 17 & 16 & 16 & 15 & 14 & 13 & 13 & 12 & 11 & 11 & 10 & 10 & 9 & 9 & 8 & 8 \\
\hline \multirow[t]{2}{*}{ Tamil Nadu } & \multirow[t]{2}{*}{$(0,1,1)$} & LCL & 11 & 10 & 9 & 8 & 7 & 7 & 6 & 6 & 5 & 5 & 4 & 4 & 4 & 3 & 3 & 3 \\
\hline & & UCL & 21 & 21 & 21 & 21 & 21 & 20 & 20 & 20 & 19 & 19 & 18 & 18 & 17 & 17 & 16 & 16 \\
\hline \multirow{3}{*}{ Uttar Pradesh } & \multirow{3}{*}{$(0,1,1)$} & Predicted & 43 & 41 & 40 & 38 & 37 & 36 & 35 & 33 & 32 & 31 & 30 & 29 & 28 & 27 & 26 & 25 \\
\hline & & LCL & 35 & 33 & 31 & 29 & 27 & 26 & 25 & 23 & 22 & 21 & 20 & 19 & 18 & 17 & 16 & 16 \\
\hline & & UCL & 53 & 52 & 52 & 51 & 50 & 50 & 49 & 48 & 47 & 46 & 45 & 44 & 44 & 43 & 42 & 41 \\
\hline \multirow{3}{*}{ West Bengal } & & Predicted & 25 & 24 & 23 & 23 & 22 & 21 & 21 & 20 & 20 & 19 & 18 & 18 & 17 & 17 & 16 & 16 \\
\hline & $(0,1,1)$ & LCL & 18 & 17 & 16 & 15 & 15 & 14 & 13 & 12 & 12 & 11 & 11 & 10 & 10 & 9 & 9 & 8 \\
\hline & & UCL & 34 & 34 & 34 & 34 & 33 & 33 & 33 & 33 & 32 & 32 & 31 & 31 & 31 & 30 & 30 & 29 \\
\hline & & Predicted & 27 & 26 & 26 & 25 & 24 & 23 & 22 & 21 & 21 & 20 & 19 & 18 & 18 & 17 & 16 & 16 \\
\hline India & $(0,1,1)$ & LCL & 24 & 22 & 21 & 20 & 19 & 18 & 17 & 16 & 15 & 14 & 14 & 13 & 12 & 12 & 11 & 11 \\
\hline & & UCL & 32 & 32 & 31 & 31 & 30 & 30 & 29 & 28 & 28 & 27 & 26 & 26 & 25 & 25 & 24 & 23 \\
\hline
\end{tabular}

NOTE: ARIMA-Autoregressive integrated moving averages; LCL—lower confidence level; UCL—upper confidence level. The states Bihar, Uttar Pradesh and Madhya Pradesh include Jharkhand, Uttarakhand and Chhattisgarh respectively. ARIMA ( $p, d, q)-p, d$ and q denote orders of auto-regression, integration (differencing) and moving average respectively. 
Table 3. (a) Predicted values of neonatal mortality rates (total) and associated $95 \%$ confidence intervals using ARIMA model by states and India, 2015-2030; (b) Predicted values of neonatal mortality rates (Rural) and associated 95\% confidence intervals using ARIMA model by states and India, 2015-2030; (c) Predicted values of neonatal mortality rates (Urban) and associated 95\% confidence intervals using ARIMA model by states and India, 2015-2030.

(a)

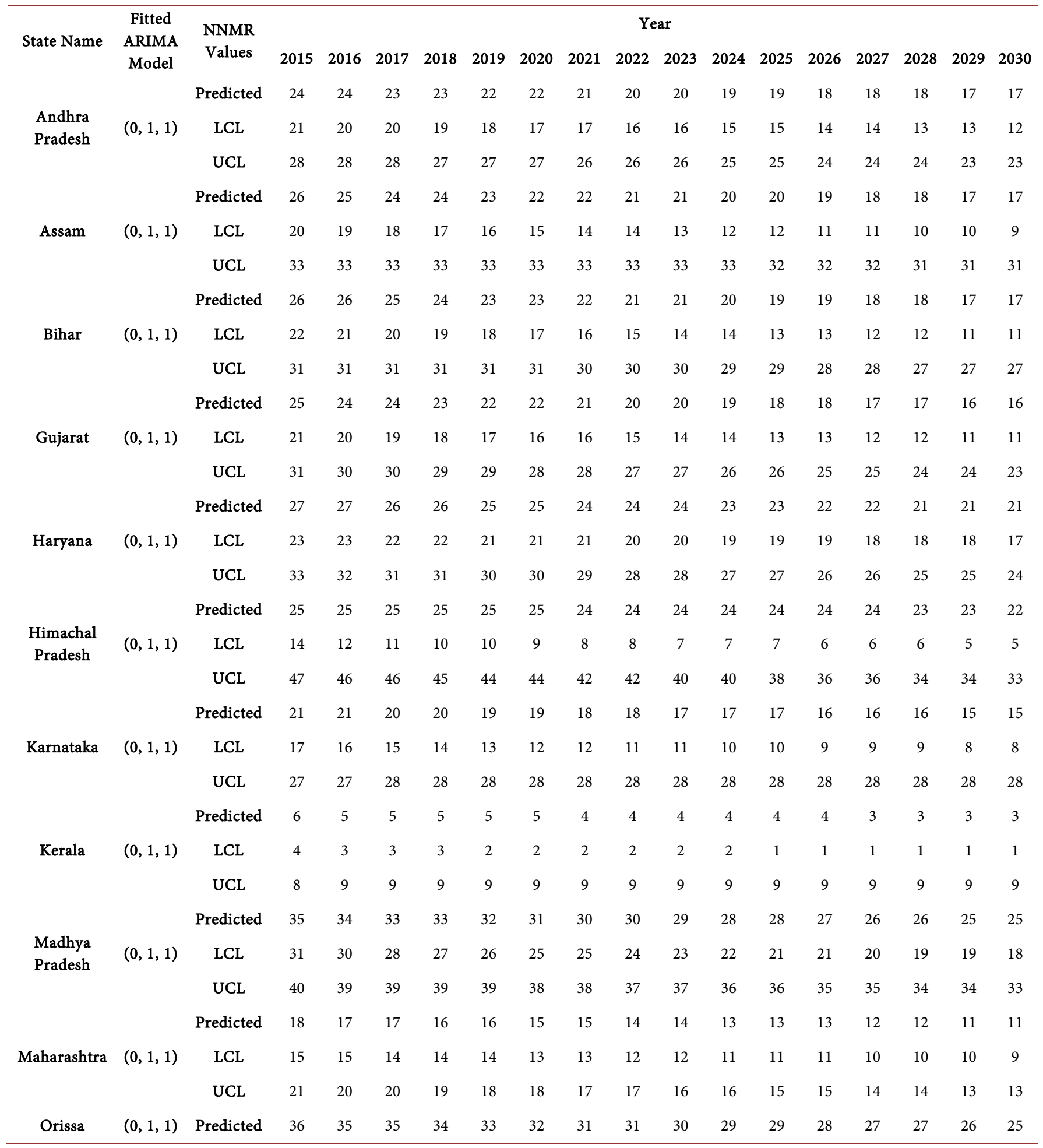


Continued

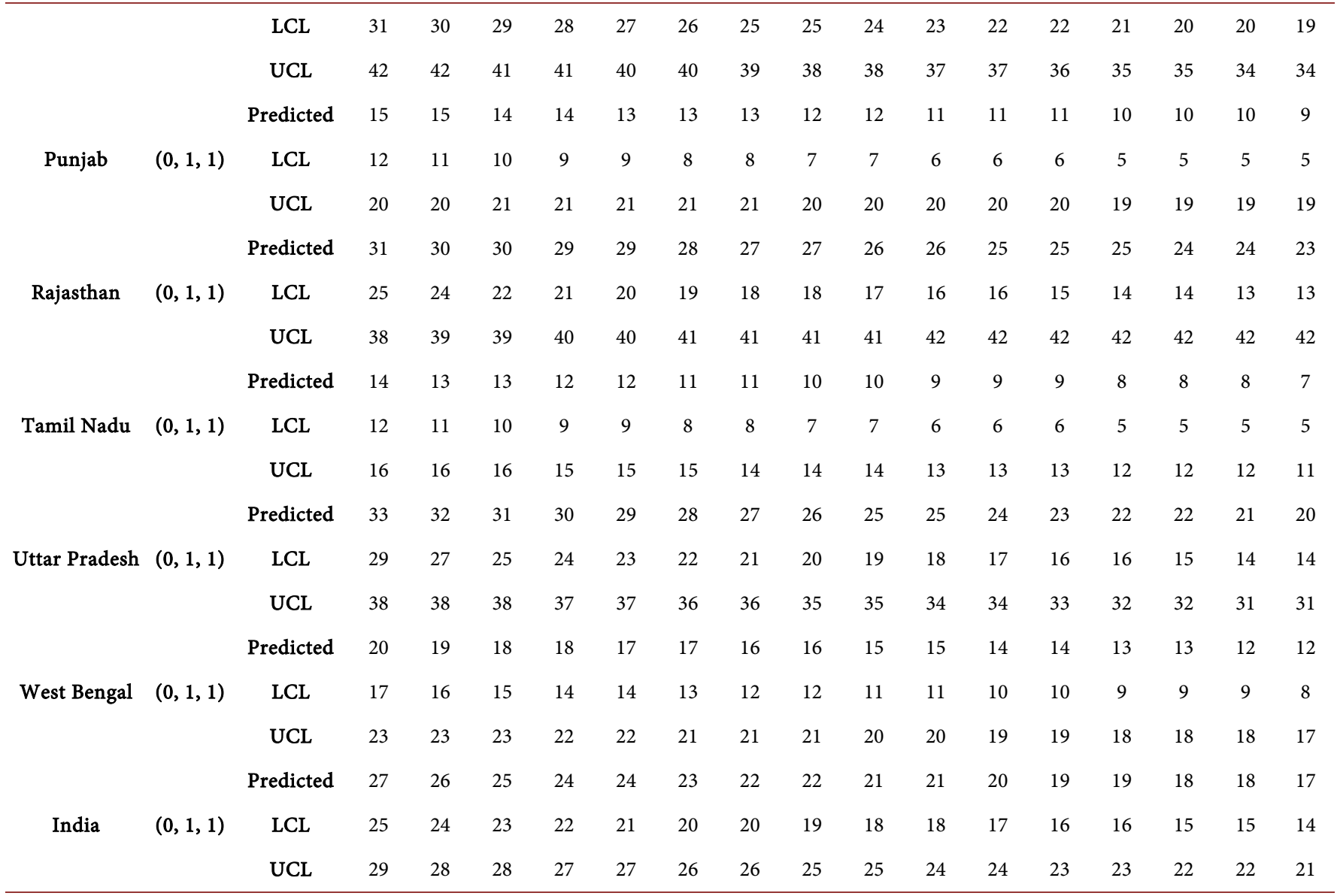

NOTE: ARIMA-Autoregressive integrated moving averages; LCL-lower confidence level; UCL-upper confidence level. The states Bihar, Uttar Pradesh and Madhya Pradesh include Jharkhand, Uttarakhand and Chhattisgarh respectively. ARIMA ( $\mathrm{p}, \mathrm{d}, \mathrm{q})-\mathrm{p}, \mathrm{d}$ and $\mathrm{q}$ denote orders of auto-regression, integration (differencing) and moving average respectively.

(b)

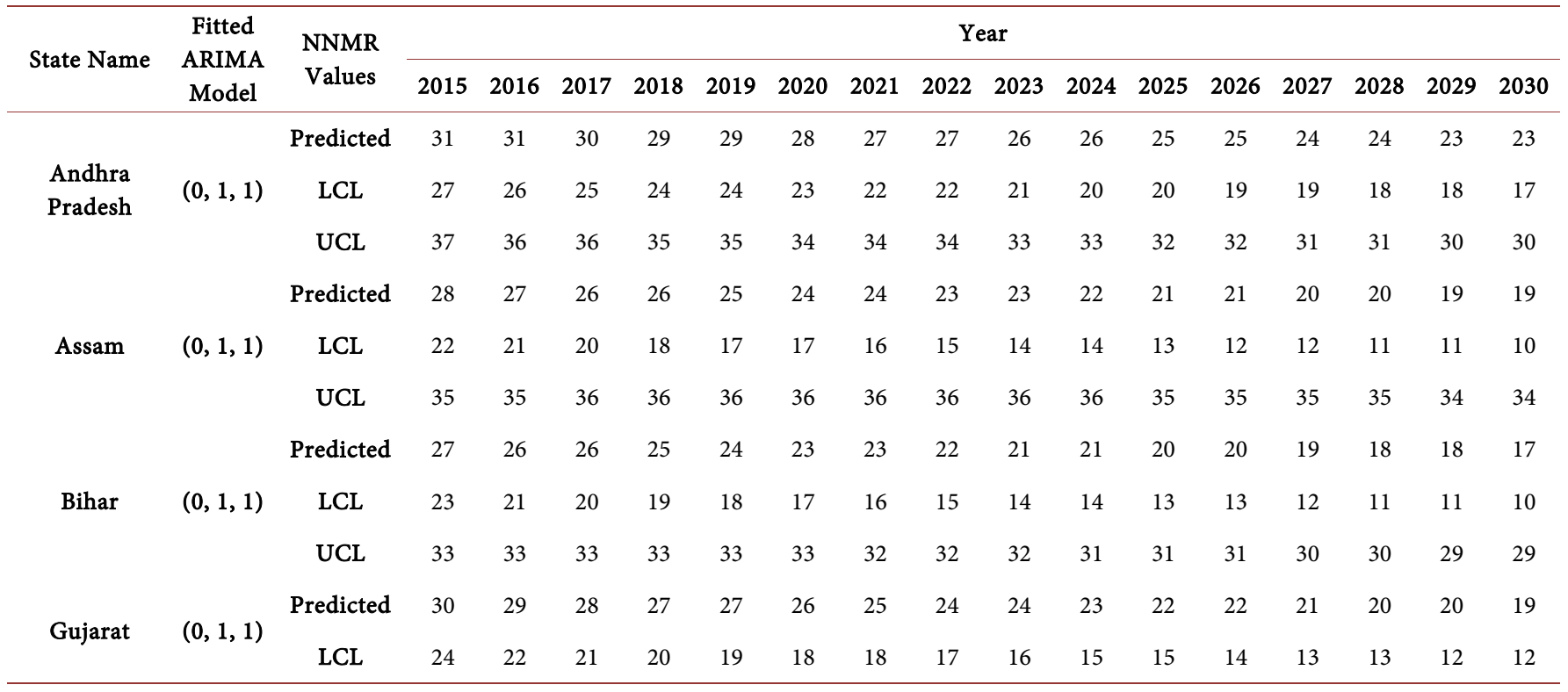




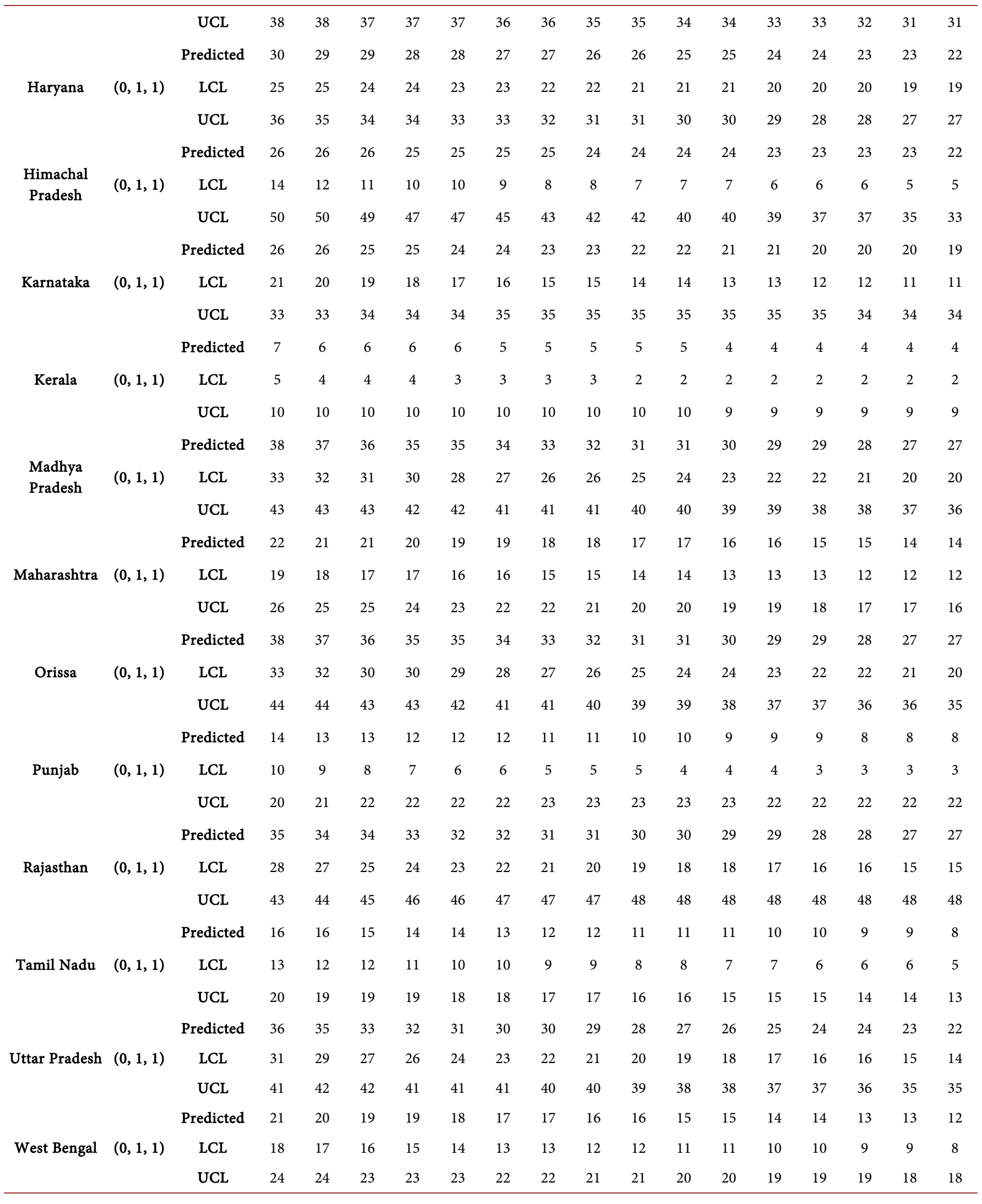


Continued

\begin{tabular}{cccccccccccccccccccc} 
& & Predicted & 30 & 29 & 28 & 27 & 26 & 26 & 25 & 24 & 24 & 23 & 22 & 22 & 21 & 21 & 20 & 20 \\
India & $(0,1,1)$ & LCL & 27 & 26 & 25 & 24 & 24 & 23 & 22 & 21 & 20 & 20 & 19 & 18 & 18 & 17 & 17 & 16 \\
& & UCL & 32 & 31 & 31 & 30 & 30 & 29 & 29 & 28 & 28 & 27 & 26 & 26 & 25 & 25 & 24 & 24 \\
\hline
\end{tabular}

NOTE: ARIMA-Autoregressive integrated moving averages; LCL—-lower confidence level; UCL—upper confidence level. The states Bihar, Uttar Pradesh and Madhya Pradesh include Jharkhand, Uttarakhand and Chhattisgarh respectively. ARIMA ( $\mathrm{p}, \mathrm{d}, \mathrm{q}$ ) $-\mathrm{p}, \mathrm{d}$ and $\mathrm{q}$ denote orders of auto-regression, integration (differencing) and moving average respectively.

(c)

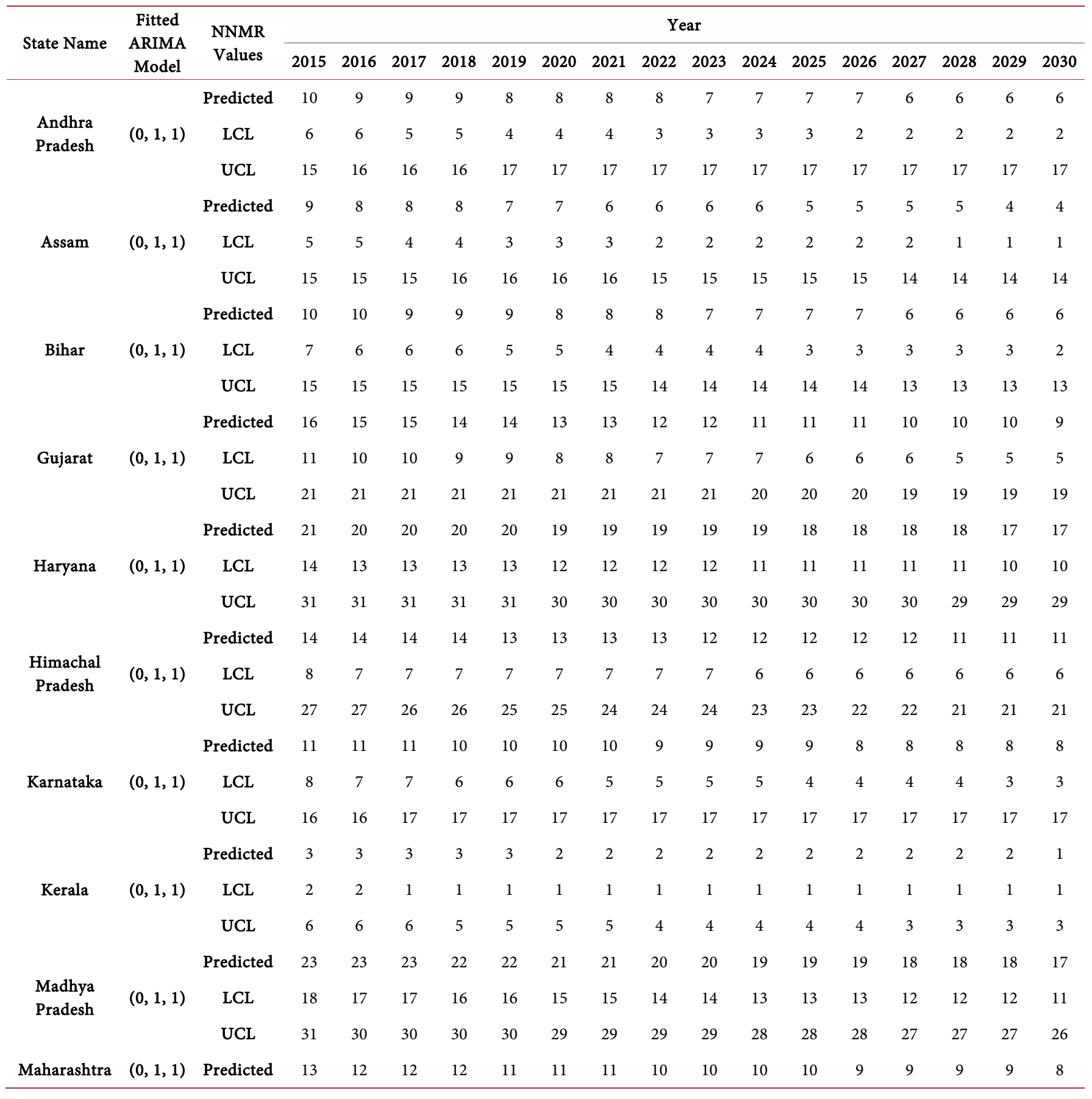




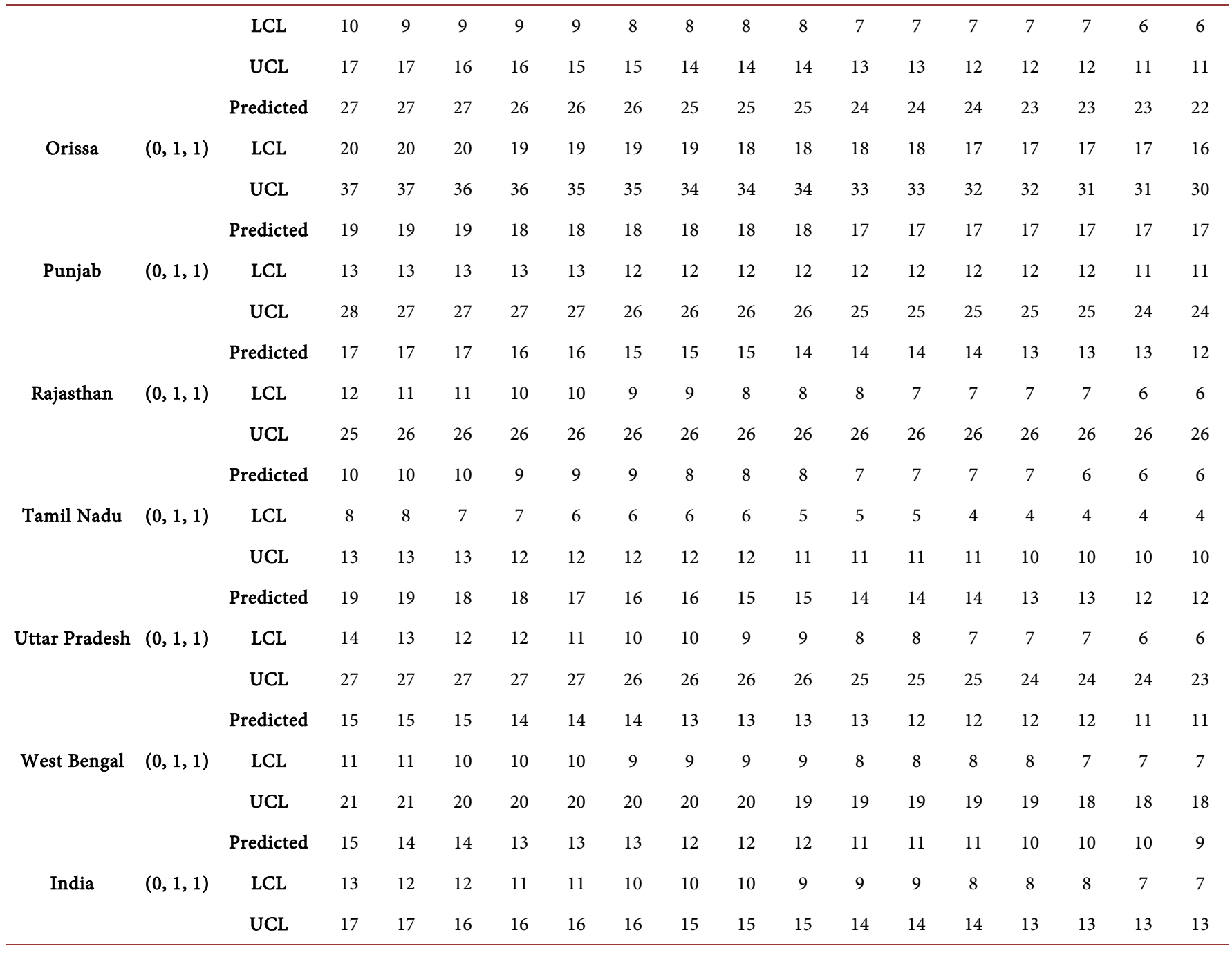

NOTE: ARIMA-Autoregressive integrated moving averages; LCL—lower confidence level; UCL-upper confidence level. The states Bihar, Uttar Pradesh and Madhya Pradesh include Jharkhand, Uttarakhand and Chhattisgarh respectively. ARIMA ( $p, d, q)-p, d$ and q denote orders of auto-regression, integration (differencing) and moving average respectively.

mortality by 2030 to half its level in 2010. Now, based on the previous SRS data of 2010, we would expect more specifically, by 2030 , India should aiming to reduce neonatal mortality to 16.3 per 1000 live births, infant mortality to 23.5 per 1000 live birth and under-5 mortality to at least as low as 29.5 per 1000 live births. The Table 1(a) shows that, the projected IMR value would be 24 per thousand live birth on 2030 more or less fulfilling the target mentioned above. However, in rural area the projected rate is slightly higher (26) for India as a whole [Table 1(b)], but in urban area this rate would be 17 only [Table 1 (c)]. Thus, India as a whole will meet the desired goal of IMR. Similarly, in case of U5MR [presented in Tables 2(a)-(c)], the projected mortality rate of 25 per thousand live birth easily reaching the 2030's target mentioned above. For rural and urban areas of India, we would expect 28 and 16 under-five mortality rates respectively. Here also, there exists a wide gap in the projected values between rural and ur- 


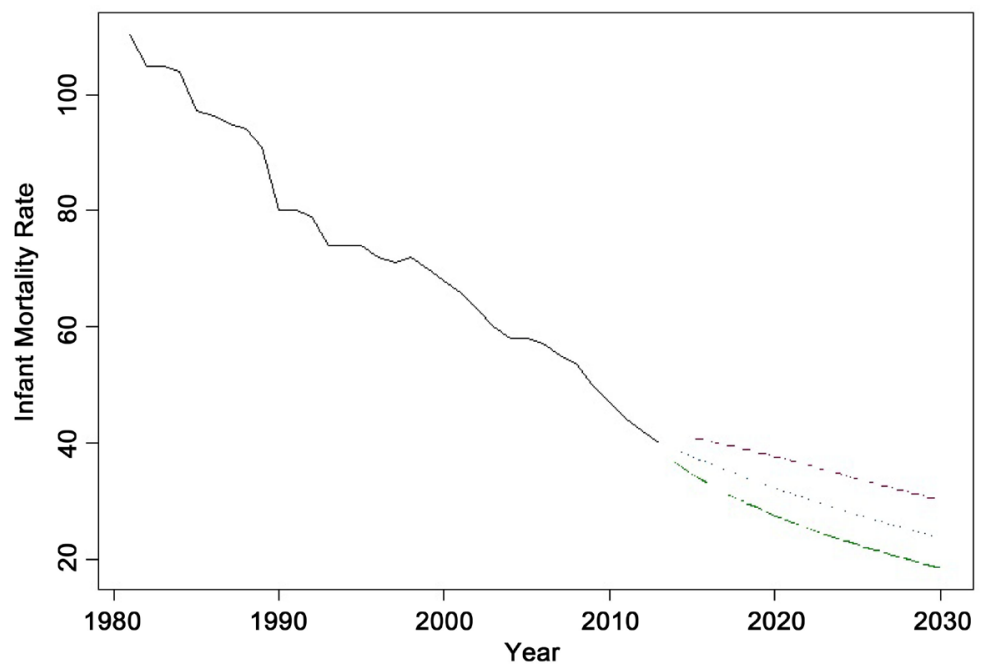

(a)

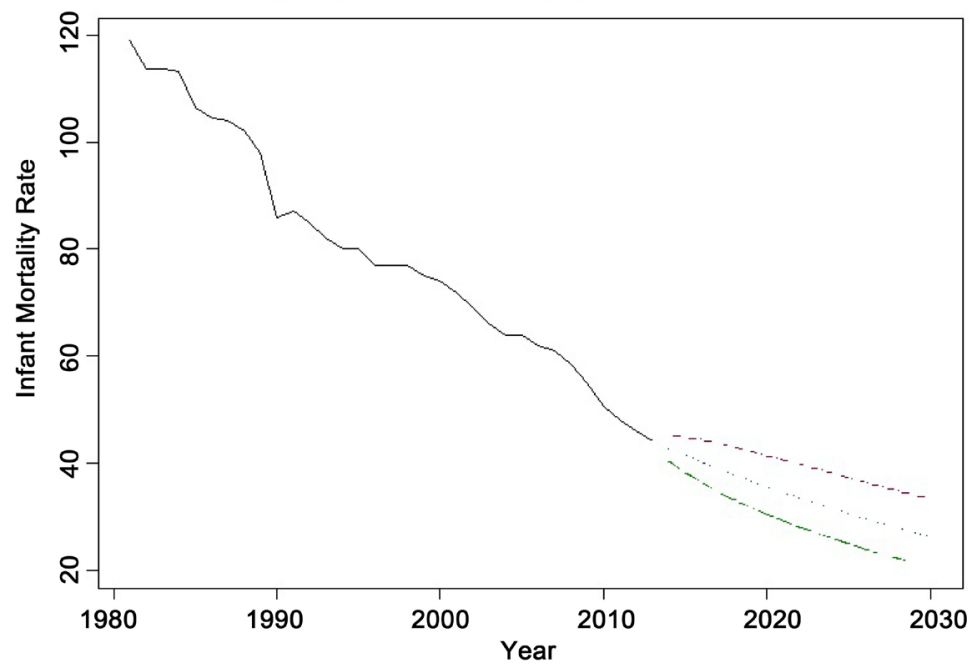

(b)

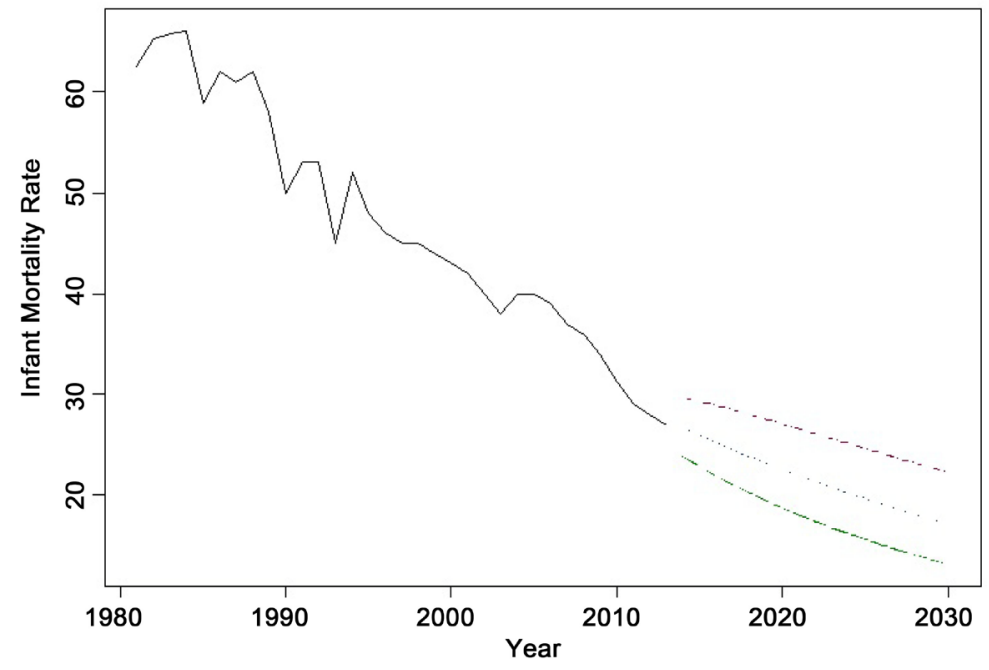

(c)

Figure 1. (a) Forecast of IMR of India (total) for the years 2015-2030; (b) Forecast of IMR of India (rural) for the years 2015-2030; (c) Forecast of IMR of India (urban) for the years 2015-2030. 

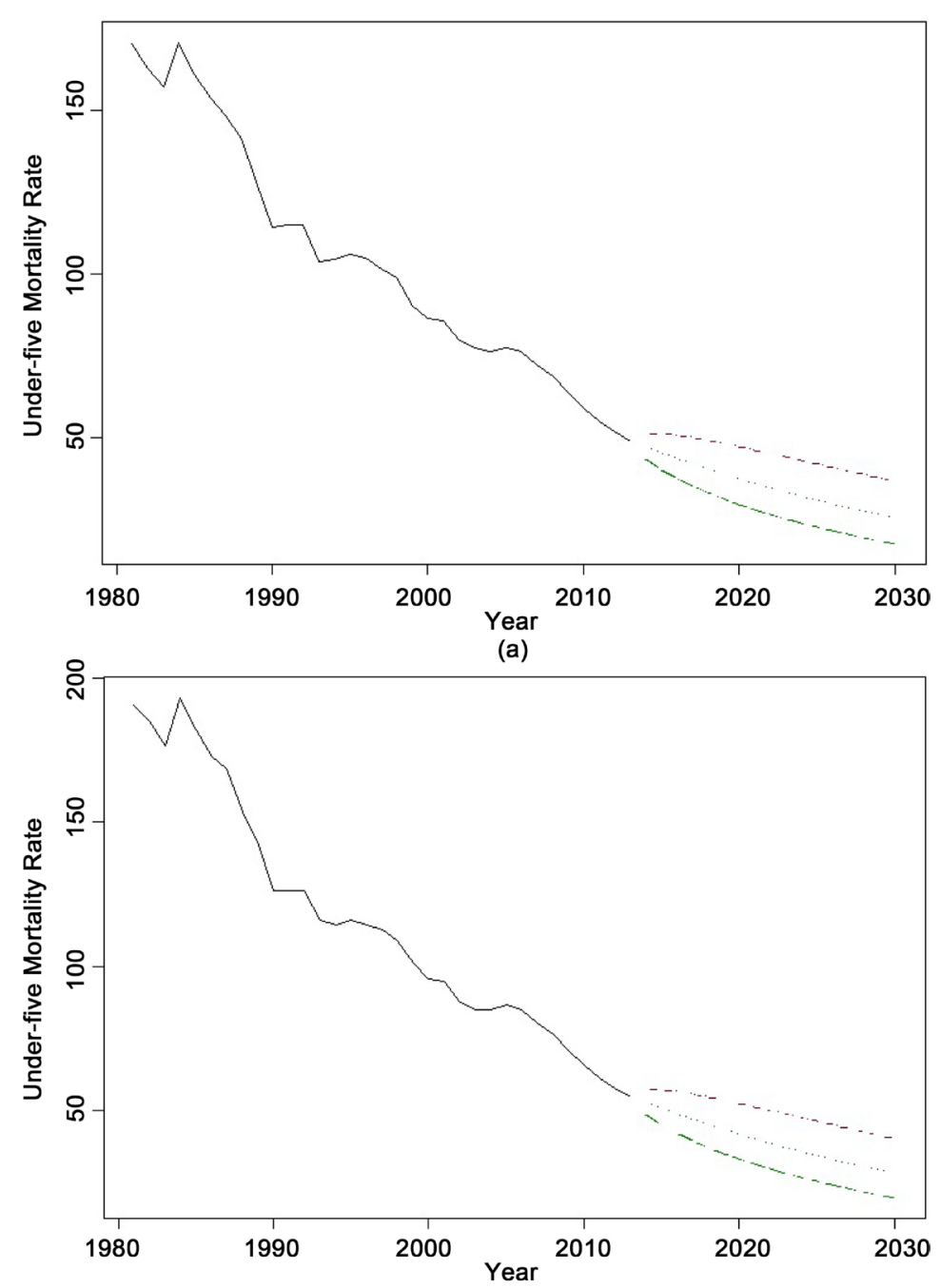

(b)

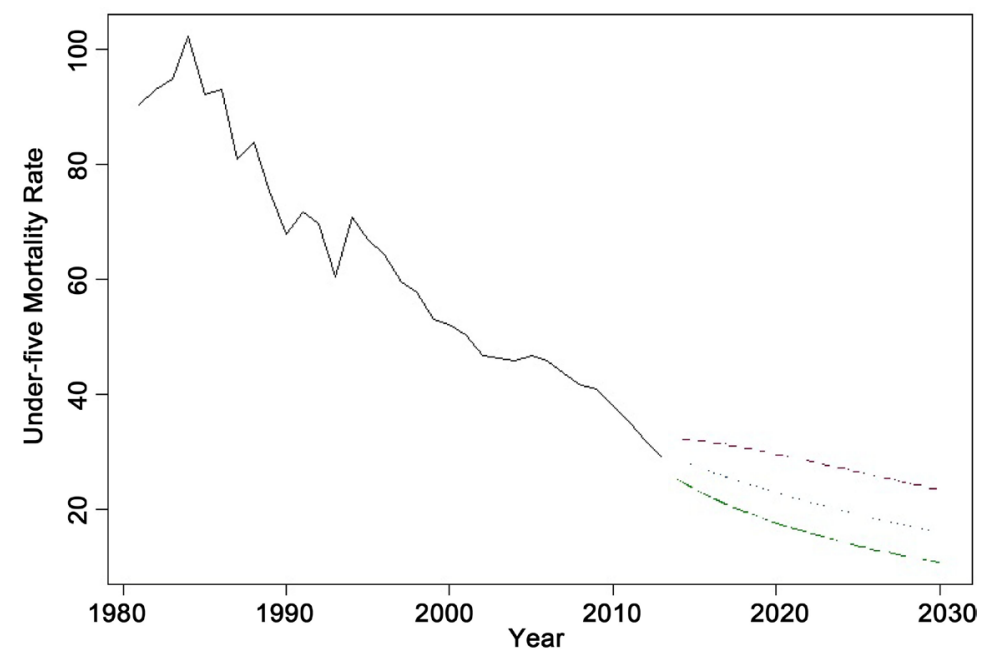

(c)

Figure 2. (a) Forecast of U5MR (total) for the years 2015-2030; (b) Forecast of U5MR of India (rural) for the years 2015-2030; (c) Forecast of U5MR of India (urban) for the years 2015-2030. 


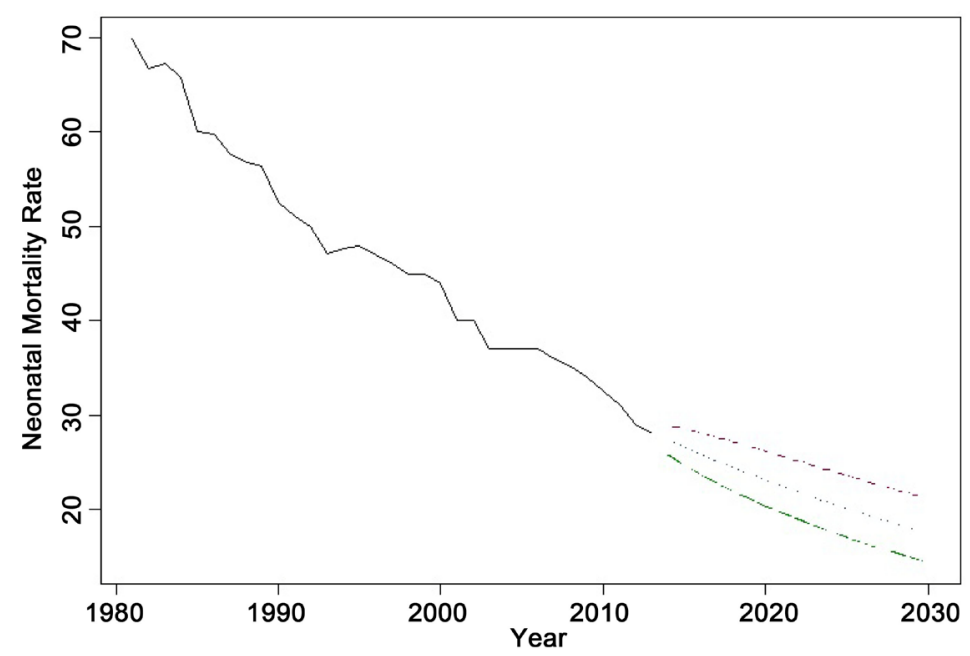

(a)

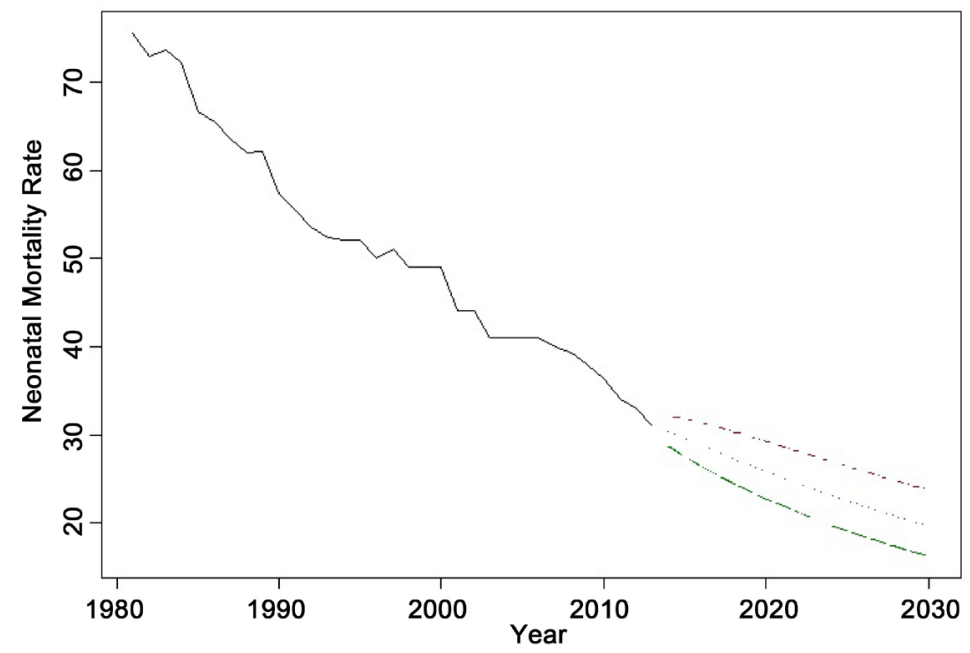

(b)

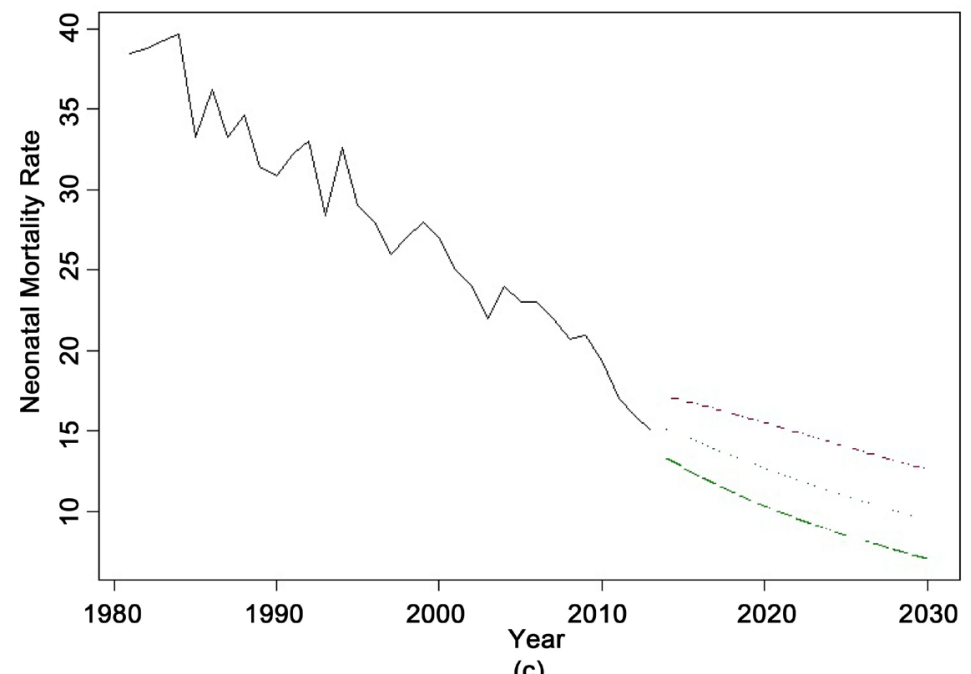

Figure 3. (a) Forecast of NNMR of India (total) for the years 2015-2030; (b) Forecast of NNMR of India (rural) for the years 2015-2030; (c) Forecast of NNMR of India (urban) for the years 2015-2030. 
ban areas of India. Now, the value of projected neonatal mortality (17) for India in the year 2030 is slightly higher than the targeted rate of 16.3. But these figures will be 20 and 9 respectively for rural and urban areas of India again indicating rural-urban divergence. Overall, in India with respect to Sustainable Development Goal-3, there is no or negligible shortfall of reaching the targets of NNMR, IMR and U5MR.The projected data in all the tables show that, India as a whole would be able to achieve the targets by 2030. But if we consider the world target of NNMR (12 per thousand live birth) and U5MR (25 per thousand live birth) mentioned earlier in the introduction section then, India will be only fulfilling the under-five mortality target.

Now, from past SRS data and projected figures [Figures 1(a)-3(c)] it may be observed, infant and under-five mortality rates are initially declining at a faster rate over time, but the rate of decline became slower from 1990's onwards. Again, reduction of IMR over time is much slower than reduction in U5MR. Moreover, the gap between the IMR and NNMR either remains same or reduced very slowly over time. Also, the NNMR itself has been declining very slowly over time. Thus it can be said that, decline in the infant mortality rate was largely due to reduction in post-neonatal mortality, with neonatal mortality rate not contributing substantially. This indicates that deaths of infants within the first four weeks have relatively greater significance in determining the level of infant and under-five mortality rates in India. As a result, currently almost two-thirds of the IMR is being contributed by the neonatal mortality rate in India. A closer look into the NNMR and U5MR projected data [Tables 2(a)-(c) and Tables $3(\mathrm{a})-(\mathrm{c})]$ show that, share of neonatal death among under-five death is increasing steadily over the future projected years. This indicates slower decline in the neonatal mortality rate than the mortality rate for older children. Moreover, projected neonatal rates indicate higher proportion of neonatal death in rural areas of India.

Let us concentrate on individual state projected values for different mortality rates. Even though India as a whole is predicted to attain SDG-3 targets, at least eight, four and six states are not predicted to attain the targeted rates for IMR, U5MR and NNMR respectively. Detailed analysis of the major states show that, Andhra Pradesh, Bihar, Gujarat, Haryana, Himachal Pradesh, Karnataka, Kerala, Maharashtra, Punjab, Rajasthan, Tamil Nadu, and West Bengal have the possibility of achieving the SDG3 goal for U5MR. In case of IMR, Gujarat, Himachal Pradesh, Karnataka, Kerala, Maharashtra, Punjab, Tamil Nadu, and West Bengal states have the prospect of attaining the target. Major shortfall of 8 points and above is observed for the states like, Assam, Madhya Pradesh and Orissa in under-five target mortality rates. Shortfall of 3 points is seen for Uttar Pradesh state. Similar observations may be mentioned here for IMR in the selected states under study. In year 2030, the states like, Gujarat, Karnataka, Kerala, Maharashtra, Punjab, Tamil Nadu and West Bengal can achieve all the NNMR, IMR and U5MR targets of sustainable Development Goal 3. According to the previous report of Millennium Development Goals by India (Country Report 2014), the major States like, Bihar, Jharkhand, Madhya Pradesh, Chhattisgarh, Orissa, Uttar Pradesh and Uttarakhand, which are also the more populated states, are among the lagging states in reduc- 
ing the poverty and not possibly able to achieve their target of halving the poverty ratio of 1990 by 2015 [1]. These States along with Maharashtra had about 193.5 million of people below poverty line in 2004-05 (64\% of total below poverty line (BPL) population) and are expected to have nearly 198 million people below poverty line in 2015 (71\% of total projected BPL population) [17]. So these heartland states of India along with Assam, Orissa and Rajasthan are also behind in effectively reducing neonatal, infant and under-five mortality rates. It is to be noted that during the projected years (Table 3(a)) the possibility of reducing neonatal deaths is very minimum in the backward states. Particularly in these backward states rural-urban differential of neonatal death will be very high compare to other states under consideration.

It has already been mentioned that, along with overall India and state wise projections of IMR, U5MR and NNMR, the present study also projected the same for rural and urban areas of each state separately. The U5MR, IMR and NNMR rates are quite very high in rural areas compared to urban areas over the years. The rural-urban differential of under-five and infant mortalities has decreased very rapidly till 1990, but after that the gap is more or less constant till 2008 and then has started to decline very slowly over the years. The rural-urban differential reduced over time may be due to the intervention of different health care and developmental programmes in different states of India. However, still there exists a constant difference due to more socio-economic and infrastructural benefits enjoyed by the urban sector compared to the rural sector in the country.

In case of under-five mortality, the states of Assam, Bihar, Madhya Pradesh, Orissa and Uttar Pradesh future projected values of 'total' and 'rural' areas are mostly coinciding throughout the projected period indicating the importance of rural child death in total death. For the states like, Punjab, Tamil Nadu, Karnataka and West Bengal, the future values for "total", "rural" and "urban" are converging rapidly over the future years. These states were also the achiever of MDG4 target in the year 2015. However, Bihar shows convergence over the projected years. So we can expect rapid improvement of U5MR in future years for this state. In both the cases of NNMR and U5MR for Assam, Madhya Pradesh, Orissa and Rajasthan, a high difference in values has been observed between the rural and urban areas over the projected years.

\section{Discussion}

It may be concluded from the foregoing results that without appropriate intervention, most of the India's backward and heartland states will not be able to reach the SDG3 target by 2030. Achievement of the targeted level will require further acceleration in the reduction of the U5MR and NNMR particularly in the highest burden states like, Assam, Madhya Pradesh (with Chhattisgarh), Orissa, Rajasthan and Uttar Pradesh (with Uttarakhand).

Particularly in the backward states extra efforts and resources will be needed to achieve reductions in under-five as well as neonatal mortality levels. However, further reductions in under-five mortality rates, to a large extent, depend on reducing the neonatal mortality. The focus now should be on neonatal mortality that has experienced 
hardly any improvement over the years. New tools and resources are needed to prevent these deaths. Thus, appropriate explanation and also intervention strategies are needed to overcome such a situation, which obstruct the policy maker in achieving the targeted goals. To address the issues of higher neonatal and early neonatal mortality, facility based newborn care services at health facilities should be established with high priority. Infrastructure strengthening, logistics and capacity building of Health workers must be ensured at the earliest for immediate intervention [1]. It has already been mentioned that, the rates for the components of under-five mortality are quite high in rural areas compared to urban areas over the years and the gap is consistent and continued over the years. Effective health care and developmental programmes are needed in the rural sector to reduce the gap in socio-economic and infrastructural benefits enjoyed by the urban sector compared to the rural sector in economy.

The relatively unfavorable international standing of India with respect to attainment of MDGs and SDGs in terms of under-five and neonatal mortalities mainly originates from the existence of substantial disparity in child survival and associated socioeconomic inequality in the country [18]. Factors contributing to the apparent stagnation and thereafter slowing decline of under-five mortality and its components include the lower socio-economic, cultural and health status of women and children in India [19]. Inequality in the use of health care services among the states also creates large differentials in U5MR components. Therefore, the implication of the present research is that government and other national and international organizations need to increase their efforts in reducing NNMR and U5MR, which calls for adjustments in planning and funding immediately. Thus, for the present $12^{\text {th }}$ Five-year plan, strategic planning and effective implementation to the matter is needed. Particularly in the heartland states immediate mass investments in building basic health infrastructure are needed in rural areas to meet up with the goals. These states are extremely lacking in reproductive and child health services in the remote village areas. Finally, poverty eradication which is also a goal among the other SDGs needs appropriate household mapping and targeting irrespective of caste and community to reduce the burden of child death.

The present comprehensive analysis of the past trends, present status, and future course of mortality among children are not only vital for developing effective maternal and child health programs and policies but is crucial in the formulation of overall national health planning. In the present study a systematic effort has been done to bring together a discussion of long-term trends and differentials in child mortality in rural and urban areas of the country based on the available time series data of different states in India. However, the present mortality forecast model particularly for the children may be improved by in-depth understanding of the various causes and predictors of mortality. This may be done by inclusion of important dependent variables like, education, gender, poverty level etc. in the analysis. This can improve the accuracy of the projection. However, the inclusion of number of variables should not be large so that confusion and complications arise in using the mortality projections. To reduce this gap in future years much attention is needed in the rural sector for states which will improve the 
neonatal and under-five mortalities. This implies burden in neonatal mortality is mostly borne by the rural sectors in these states and needs appropriate attention.

At the end it may be mentioned that we have included the latest data of neonatal mortality (NNMR), infant mortality (IMR) and under-five mortality (U5MR) rates from India's Sample Registration System (SRS). Hence, our interpretation relied completely on the quality of SRS data. An evaluation of SRS data exhibited omission rates of $1.8 \%$ for births and $2.5 \%$ for deaths [20]. Towards the method, there have been some research indicating that ARIMA time series modeling may be more suitable than the simple trend fitting approach, which suffers from model specification error [21]. Particularly, researchers have shown that the forecasting methodology can be improved by incorporating the ARIMA method [22]. However, use of ARIMA model for future forecasting and interpretation of the results requires caution because stationary time series system, which passes through different stages of development during the period under study, may also experience variations in coverage which can affect the quality of time series.

\section{Conclusions}

It may be concluded from the foregoing analysis that without immediate intervention, India's majority backward and heartland states would not be able to achieve the SDG3 target by 2030. Achievement of the targeted level will require further acceleration in the reduction of the U5MR and NNMR particularly in the highest burden states like, Assam, Madhya Pradesh (with Chhattisgarh), Orissa, Rajasthan, Uttar Pradesh (with Uttarakhand), and Bihar (with Jharkhand).

Further reductions in infant and under-five mortality rates, to a large extent, depend on reducing the neonatal mortality. Hence, focus should be on neonatal mortality that has hardly shown any improvement so far. New tools and resources are needed to prevent these deaths. Appropriate intervention strategies are needed to overcome such a situation, which obstruct the policy maker to achieve the targeted goals. To address the issues of higher neonatal and early neonatal mortality, facility based newborn care services at health facilities should be established with high priority. Infrastructure strengthening, logistics and capacity building of Health workers must be ensured at the earliest for immediate intervention. Effective health care and developmental programmes are needed in the rural sector to reduce the gap in socio-economic and infrastructural benefits enjoyed by the urban sector compared to the rural sector in economy.

The current comprehensive analysis of the past trends, present status, and future course of mortality among children is not only vital for developing effective maternal and child health programmes and policies but also crucial in the formulation of overall national health planning.

\section{Strengths and Limitations of This Study}

\subsection{Strengths}

- The child mortality is a key element of achieving new targets for SDGs for India and its states. 
- It is prudent to forecast the child mortality and its components assessing the recent interventions in reducing such deaths.

- The present paper has used autoregressive integrated moving average (ARIMA) time series model to forecast the neonatal, infant and under-five mortalities in India for the period of 2015-2030 by using data from the Sample Registration System (SRS) for the period 1981-2013.

- The projection showed that India as a whole would be able to achieve the SDG targets of NNMR, IMR and U5MR by 2030.

\subsection{Limitation}

- The ARIMA model, as all forecasting methods, is essentially "backward lookinge", such that, the long term forecast eventually goes to be straight line at predicting series with turning points.

\section{Competing Interests}

The authors declare that they have no competing interests.

\section{Author's Contributions}

$\mathrm{PD}, \mathrm{AP}, \mathrm{DS}$ and BKG conceived and design the study, $\mathrm{PD}$ and AKS contributed in the development of method and analysis of data, PM, RGM and NC drafted the manuscript. All authors contributed to the interpretation of results and critical review towards finalization of manuscript.

\section{Data Sharing Statement}

Data from Sample Registration System is available in public domain (www.censusindia.gov.in).

\section{References}

[1] Ministry of Statistics and Programme Implementation (MOSPI) (2014) Millennium Development Goals-India Country Report 2014. Social Statistics Division, (MOSPI), Government of India.

[2] UNICEF, WHO, WORLD BANK GROUP and UN (2015) Levels \& Trends in Child Mortality: Report 2015, Estimates Developed by the UN Inter-Agency Group for Child mortality Estimation. New York.

[3] UNICEF (2014) The State of the World's Children 2015: Reimagine the Future: Innovation for Every Child. United Nations Children's Fund (UNICEF), New York.

[4] Sample Registration System (SRS) (2013) Sample Registration System Statistical Report. Office of the Registrar General, India, Ministry of Home Affairs, Government of India, New Delhi.

[5] UN (United Nations) (2016) Sustainable Development Goals: 17 Goals to Transform Our Worlds. New York. http://www.un.org/sustainabledevelopment/

[6] United Nations (2013) The Millennium Development Goals Report 2013. New York.

[7] World Bank (2005) Attaining the Millennium Development Goals in Bangladesh. World 
Bank, Washington DC.

[8] World Bank (2005) Attaining the Millennium Goals in Sri Lanka: How Likely and What Will It Take To Reduce Poverty, Child Mortality and Malnutrition, and to Increase School Enrollment and Completion? South Asia Region: 63, World Bank, Human Development Unit, Washington DC.

[9] Reddy, H., Pradhan, M.R., Ghosh, R. and Khan, A.G. (2012) India's Progress towards the Millennium Development Goals 4 and 5 on Infant Mortality. WHO South-East Asia Journal of Public Health, 1, 279-289.

[10] World Health Organization (2006) Neonatal and Perinatal Mortality-Country, Regional and Global Estimates 2004. Department of Making Pregnancy Safer (MPS), WHO, Geneva.

[11] Chandhoik, N., Sahu, D., Gulati, B.K., Shukla, A.K. and Pandey, A. (2016) Estimates of Infant and Child Mortality in India: A Comparison between SRS and NFHS. In: Sharma, S., Ed., Demographic Challenges in India, Athena Academic, London, 135-160.

[12] Johnathan, M. (2006) Modeling Risk: Applying Monte Carlo Simulation, Real Options Analysis, Forecasting and Optimization Techniques. Wiley Finance Series, John Wiley \& Sons Inc., Hoboken.

[13] Myer, G.L. and Yanagida, J.F. (1984) Combining Annual Econometric Forecasts with Quarterly ARIMA Forecasts: A Heuristic Approach. Western Journal of Agricultural Economics, 9, 200-206.

[14] Box, G.E.P. and Jenkins, G.M. (1976) Time Series Analysis: Forecasting and Control. Holden Day, San Francisco.

[15] O’Neill B.C., Balk, D., Brickman, M. and Ezra, M. (2001) A Guide to Global Population Projections. Demographic Research, 4, 203-288. https://doi.org/10.4054/DemRes.2001.4.8

[16] Box, G.E.P., Jenkins, G.M. and Reinsel, G.C. (1994) Time Series Analysis, Forecasting and Control. 3rd Edition, Prentice Hall, Englewood Cliffs.

[17] Central Statistical Organization, Ministry of Statistics and Programme Implementation (2009) Millennium Development Goals-India Country Report 2009. Mid-Term Statistical Appraisal.

[18] De, P. and Dhar, A. (2013) Inequality in Child Mortality across Different States of India: A Comparative Study. Journal of Child Health Care, 17, 397-409. https://doi.org/10.1177/1367493512468359

[19] De, P. and Dhar, A. (2009) Deprivation and Neonatal Mortality: An Approach to Evaluate Reasons behind the Deprivation Leading to Mortality. In: Singh, K.K., Yadav, R.C. and Pandey, A., Eds., Population, Poverty \& Health-Analytical Approaches, National Institute of Medical Statistics, Hindustan Publishing Corporation (India), New Delhi, 111-131.

[20] Registrar General of India (1988) Report on Intensive Enquiry Conducted in Sub-Sample of SRS Units. Occasional Paper No. 1, Office of the Registrar General of India, Ministry of home Affairs, New Delhi.

[21] Farmer, R.D. and Emami, J. (1990) Models for Forecasting Hospital Bed Requirements in the Acute Sector. Journal of Epidemiology \& Community Health, 44, 307-312. https://doi.org/10.1136/jech.44.4.307

[22] Milner, P.C. (1997) Ten-Year Follow-Up of ARIMA Forecasts of Attendances at Accident and Emergency Departments in the Trent Region. Statistics in Medicine, 16, 2117-2125. https://doi.org/10.1002/(SICI)1097-0258(19970930)16:18<2117::AID-SIM649>3.0.CO;2-E 
Submit or recommend next manuscript to SCIRP and we will provide best service for you:

Accepting pre-submission inquiries through Email, Facebook, LinkedIn, Twitter, etc.

A wide selection of journals (inclusive of 9 subjects, more than 200 journals)

Providing 24-hour high-quality service

User-friendly online submission system

Fair and swift peer-review system

Efficient typesetting and proofreading procedure

Display of the result of downloads and visits, as well as the number of cited articles

Maximum dissemination of your research work

Submit your manuscript at: http://papersubmission.scirp.org/

Or contact health@scirp.org 